This work is licensed under a Creative Commons Attribution License (CC BY 4.0).

Research article

urn:1sid:zoobank.org:pub:D0B71B76-0375-408A-B962-C33B714E3EF4

\title{
Two new Cyrtodactylus (Squamata, Gekkonidae) from Northeast India
}

\author{
Jayaditya PURKAYASTHA ${ }^{\circledR 1}$, Hmar Tlawmte LALREMSANGA ${ }^{\circledR 2, *}$, \\ Beirathie LITHO ${ }^{\circledR 3}$, Yashpal Singh RATHEE ${ }^{\circledR 4}$, Sanath Chandra BOHRA ${ }^{\oplus 5}$, \\ Vabeiryureilai MATHIPI ${ }^{\circledR 6}$, Lal BIAKZUALA ${ }^{\circledR 7}$ \& Lal MUANSANGA $^{\circledR 8}$ \\ ${ }^{1,5}$ Help Earth, 16, RNC Path, Lachitnagar, Guwahati 781007, Assam, India. \\ 2, 6,7,8 Developmental Biology and Herpetology Laboratory, Department of Zoology, Mizoram \\ University, Aizawl 796004, Mizoram, India. \\ ${ }^{3}$ RMSA School Lobo, Lobo, 796901, Siaha District, Mizoram, India. \\ ${ }^{4}$ Herpsmitten, Umroi Military Station, Umiam 793103, Meghalaya, India. \\ *Corresponding author: htlrsa@yahoo.co.in \\ 1Email: mail.jayaditya@gmail.com \\ 3Email: lithopawhnai8@gmail.com \\ ${ }^{4}$ Email: ypsr99@gmail.com \\ ${ }^{5}$ Email: sreptilian6@gmail.com \\ ${ }^{6}$ Email: m.vabeiryureilai@gmail.com \\ ${ }^{7}$ Email: bzachawngthu123@gmail.com \\ ${ }^{8}$ Email: muanapunte16@gmail.com
}

\begin{abstract}
${ }^{1}$ urn:1sid:zoobank.org:author:44FA413E-CA6A-4306-BCFA-1D97C10CE6E3
${ }^{2}$ urn:lsid:zoobank.org:author:F7D2FBD8-87EB-4962-BC8E-87AD754F7E8C

${ }^{3}$ urn:lsid:zoobank.org:author:4F95090B-0905-4E74-A1BE-CD2666E6AA60

${ }^{4}$ urn:1sid:zoobank.org:author:C30DA0D2-9F6E-4931-820F-E1F98DB948F8

${ }^{5}$ urn:1sid:zoobank.org:author:2431BB13-19D4-4BD1-9E1C-F7E49612C774

${ }^{6}$ urn:1sid:zoobank.org:author:60A28A8C-8991-4249-BD86-10809ED3AF80

${ }^{7}$ urn:lsid:zoobank.org:author:D5DC18A0-9466-45DF-9C07-FCBC776185E4

${ }^{8}$ urn:lsid:zoobank.org:author:2DE8B361-3407-4B62-BB6B-0F60D3A54880
\end{abstract}

\begin{abstract}
We describe two new species of Cyrtodactylus Gray, 1827, each from the Indian states of Meghalaya and Mizoram based on morphology and ND2 gene sequences. The new species are a part of the Cyrtodactylus khasiensis group. Both species represent the highland clade within the south of Brahmaputra clade of Indo-Burmese Cyrtodactylus. Based on ND2 gene sequence, the species from Meghalaya have an uncorrected p-distance of $4.21 \%-4.25 \%$ from a lowland species C. guwahatiensis Agarwal, Mahony, Giri, Chaitanya \& Bauer, 2018 and is a sister taxon to C. septentrionalis Agarwal, Mahony, Giri, Chaitanya $\&$ Bauer, 2018. The species from Mizoram differ from its sister species C. bengkhuaiai Purkayastha, Lalremsanga, Bohra, Biakzuala, Decemson, Muansanga, Vabeiryureilai, Chauhan \& Rathee, 2021 by a p-distance of $8.33 \%$.
\end{abstract}

Keywords. Biodiversity, Indo-Burma Hotspot, bent-toed gecko, ND2, systematics. 
Purkayastha J., Lalremsanga H.T., Litho B., Rathee Y.S., Bohra S.C., Mathipi V., Biakzuala L. \& Muansanga L. 2022. Two new Cyrtodactylus (Squamata, Gekkonidae) from Northeast India. European Journal of Taxonomy 794 : 111-139. https://doi.org/10.5852/ejt.2022.794.1659

\section{Introduction}

The genus Cyrtodactylus Gray, 1827 is represented by around 320 species worldwide and is the third most speciose vertebrate genus in the world (Grismer et al. 2021). The members of the genus range from South Asia to Melanesia with high diversity in south Asia (Malaysia: 49 species, Vietnam: 48 species, Myanmar: 45 species, Thailand: 38 species, Indonesia: 37 species, Laos: 25 species; see Uetz et al. 2022). India is home to 38 species of Cyrtodactylus (Kamei \& Mahony 2021; Purkayastha et al. 2021; Uetz et al. 2022) of which 14 species are known from Northeast India namely C. guwahatiensis Agarwal, Mahony, Giri, Chaitanya \& Bauer, 2018 (Assam); C. jaintiaensis Agarwal, Mahony, Giri, Chaitanya \& Bauer, 2018 (Meghalaya); C. kazirangaensis Agarwal, Mahony, Giri, Chaitanya \& Bauer, 2018 (Assam); C. khasiensis (Jerdon, 1870) (historically reported from throughout Northeast India but is known with certainty only from East Khasi Hills district, Meghalaya; see Agarwal et al. 2018a); C. montanus Agarwal, Mahony, Giri, Chaitanya \& Bauer, 2018 (Tripura and Mizoram); C. nagalandensis Agarwal, Mahony, Giri, Chaitanya \& Bauer, 2018 (Nagaland); C. septentrionalis Agarwal, Mahony, Giri, Chaitanya \& Bauer, 2018 (Assam); C. tripuraensis Agarwal, Mahony, Giri, Chaitanya \& Bauer, 2018 (Tripura); C. urbanus Purkayastha, Das, Bohra, Bauer \& Agarwal, 2020 (Assam and Meghalaya); C. karsticola Purkayastha, Lalremsanga, Bohra, Biakzuala, Decemson, Muansanga, Vabeiryureilai, Chauhan \& Rathee, 2021 (Meghalaya); C. agarwali Purkayastha, Lalremsanga, Bohra, Biakzuala, Decemson, Muansanga, Vabeiryureilai, Chauhan \& Rathee, 2021 (Meghalaya); C. aaronbaueri Purkayastha, Lalremsanga, Bohra, Biakzuala, Decemson, Muansanga, Vabeiryureilai, Chauhan \& Rathee, 2021 (Mizoram), C. bengkhuaiai Purkayastha, Lalremsanga, Bohra, Biakzuala, Decemson, Muansanga, Vabeiryureilai, Chauhan \& Rathee, 2021 (Mizoram) and C. arunachalensis Mirza, Bhosale, Ansari, Phansalkar, Swant, Gowande \& Patel, 2021 (Arunachal Pradesh).. So far all the species of Cyrtodactylus described from Northeast India are members of the C. khasiensis group (Grismer et al. 2021) which may be further sub-divided into highland and lowland clades (Agarwal et al. 2018b). Again, it is presumed that the Brahmaputra river acts as a barrier for the dispersal of species and thus, most of the species described from Northeast India are part of south of the Brahmaputra clade except for the species from Arunachal Pradesh including $C$. arunachalensis. Herein, we describe a new species each from the state of Meghalaya and Mizoram (Fig. 1). Both species are part of the C. khasiensis group and members of south of Brahmaputra in the highland clade.

\section{Material and methods}

Surveys were carried out in Siaha district of Mizoram (permission no.A.33011/2/99-CWLW/225) and Ri-Bhoi district of Meghalaya (permission no.FWC/G/173/Pt-V/2377-87). Collected samples were fixed and preserved in formaldehyde solution and deposited in the National Zoological Collection, maintained by the Department of Zoology, Mizoram University, Aizawl, Mizoram, India. Measurements were made using a Mitutoyo ${ }^{\mathrm{TM}}$ dial calliper to the nearest $0.1 \mathrm{~mm}$. Measurements and scale counts were made on the right side of the specimen unless otherwise stated.

\section{Abbreviations and terminology}

The following measurements and meristics were recorded following (Agarwal et al. 2018a), abbreviations and terminology are as follows: SVL, snout to vent length; TRL, trunk length, length between axilla and groin ; BW, maximum body width; TL, tail length; TW, tail width taken on first tail segment; TD, tail depth taken on first tail segment; HL, head length from the snout tip to the retroarticular process of the jaw; HW, maximum head width; JW, jaw width taken ventrally at the retroarticular process of the jaw; $\mathrm{HD}$, maximum head depth; FL, forearm length, length between elbow and wrist; CL, crus length, length 


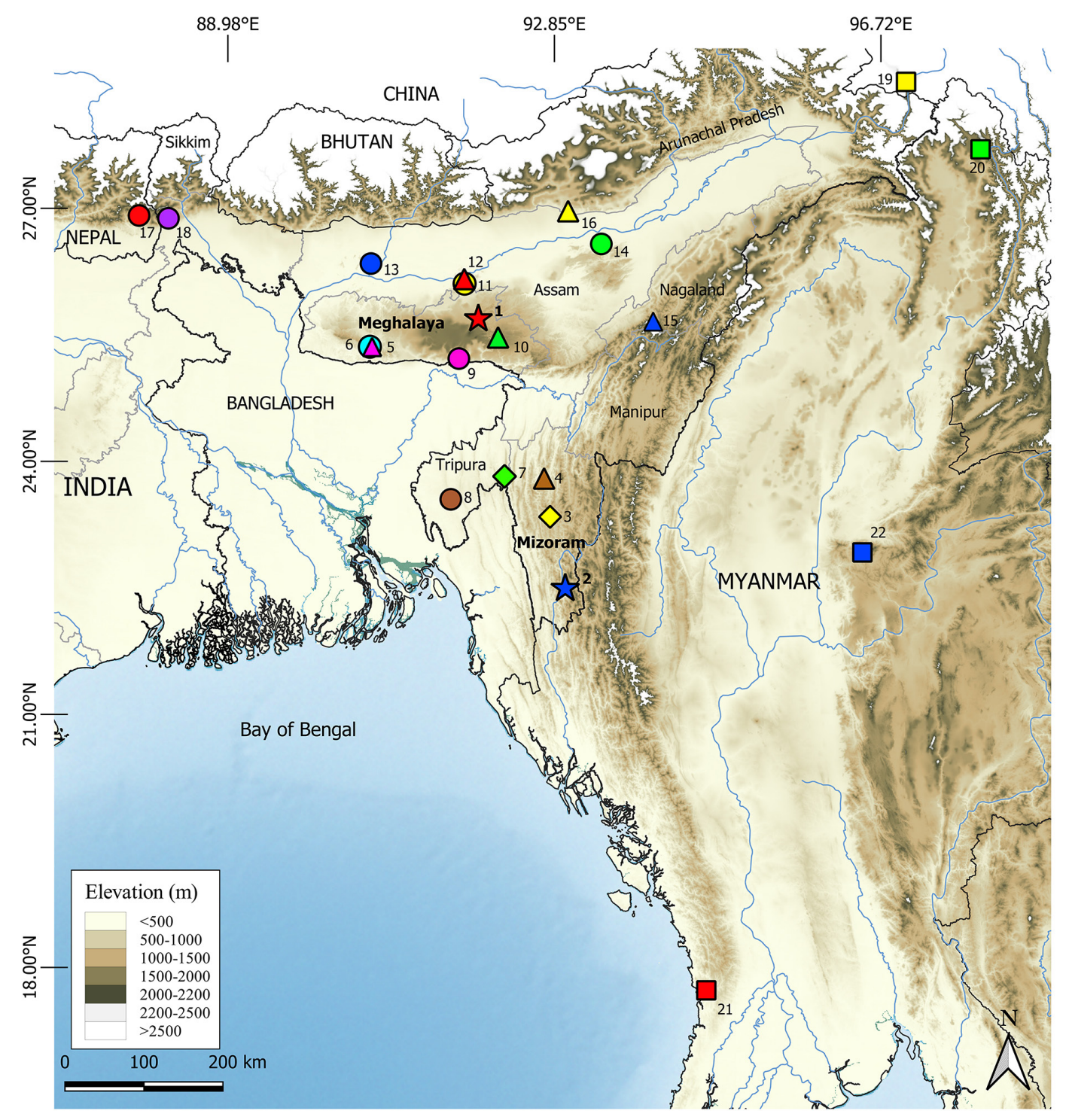

Fig. 1. Elevation map showing the type localities for the two newly described species of Cyrtodactylus and the congeners compared in this paper: 1. Cyrtodactylus exercitus sp. nov. (red star). 2. C. siahaensis sp. nov. (blue star). 3. C. bengkhuaiai Purkayastha, Lalremsanga, Bohra, Biakzuala, Decemson, Muansanga, Vabeiryureilai, Chauhan \& Rathee, 2021. 4. C. aaronbaueri Purkayastha, Lalremsanga, Bohra, Biakzuala, Decemson, Muansanga, Vabeiryureilai, Chauhan \& Rathee, 2021. 5. C. karsticola Purkayastha, Lalremsanga, Bohra, Biakzuala, Decemson, Muansanga, Vabeiryureilai, Chauhan \& Rathee, 2021. 6. C. agarwali Purkayastha, Lalremsanga, Bohra, Biakzuala, Decemson, Muansanga, Vabeiryureilai, Chauhan \& Rathee, 2021. 7. C. montanus Agarwal, Mahony, Giri, Chaitanya \& Bauer, 2018. 8. C. tripuraensis Agarwal, Mahony, Giri, Chaitanya \& Bauer, 2018. 9. C. khasiensis (Jerdon, 1870). 10. C. jaintiaensis Agarwal, Mahony, Giri, Chaitanya \& Bauer, 2018. 11. C. guwahatiensis Agarwal, Mahony, Giri, Chaitanya \& Bauer, 2018. 12. C. urbanus Purkayastha, Das, Bohra, Bauer \& Agarwal, 2020. 13. C. septentrionalis Agarwal, Mahony, Giri, Chaitanya \& Bauer, 2018. 14. C. kazirangaensis Agarwal, Mahony, Giri, Chaitanya \& Bauer, 2018. 15. C. nagalandensis Agarwal, Mahony, Giri, Chaitanya \& Bauer, 2018. 16. C. arunachalensis Mirza, Bhosale, Ansari, Phansalkar, Swant, Gowande \& Patel, 2021. 17. C. markuscombaii (Darevsky, Helfenberger, Orlov \& Shah, 1998) and C. martinstolli Darevsky, Helfenberger, Orlov \& Shah, 1998). 18. C. himalayicus (Annandale, 1906). 19. C. cayuensis Li, 2007. 20. C. tamaiensis (Smith, 1940). 21. C. ayeyarwadyensis Bauer, 2003. 22. C. mandalayensis Mahony, 2009. 
between knee and ankle; OD, orbit diameter; NO, distance between the posterior edge of the nostril and the anterior orbital border; SO, distance between the snout tip and the anterior orbital border; OE, distance between posterior orbital border and the anterior border of the ear; EL, maximum ear length; ES, anterior border of the ear to the snout tip; IN, internarial distance; IO, minimum interorbital distance between left and right supraciliary rows; RL, maximum rostral length; RW, maximum rostral width; ML, maximum mental length; MW, maximum mental width; PMIL, maximum length of inner postmental; PMIIL, maximum length of outer postmental; digits were measured from the proximal apex with the neighbouring digit to the tip (excluding the claw), and numbered from inner (I) to outer (V) as follows: on manus, FIL, FIIL, FIIIL, FIVL, FVL; on pes, TIL, TIIL, TIIIL, TIVL, TVL; PcP, precloacal pores; PcFP, precloacofemoral pores; MVSR, mid-ventral scale rows (counted between ventrolateral folds); PVT, paravertebral tubercles on the trunk only (counted between the level of the axilla and the level of the groin); DTR, dorsal tubercle rows (counted transversely across the body); SL, total supralabials; IL, total infralabials. Two separate series for subdigital lamellae were counted on all digits of the right manus and right pes, a basal series, that includes scales of a width at least twice the diameter of palmar scales up to and including a single large scale at the digital inflection, and an apical series, including lamellae distal to the digital inflection and not including the ventral claw sheath or nonlamellar scales between the basal and apical lamellae series (counted separately), abbreviated as follows: on manus, FILam, FIILam, FIIILam, FIVLam, FVLam; on pes, TILam, TIILam, TIIILam, TIVLam, TVLam.

The newly described species are compared morphologically with all known species from the khasiensis group (Agarwal et al. 2014) and other species from Northeast India and surrounding countries. Comparisons were made through examination of type specimens (see Appendix I), literature containing original descriptions and literature reviewing a species based on type specimen (i.e., Darevsky et al. 1998; Bauer 2003; Li 2007; Mahony 2009; Agarwal et al. 2018a, 2018b; Kamei \& Mahony 2021; Mirza et al. 2021; Purkayastha et al. 2020, 2021).

\section{Molecular data}

For this study, four specimens of Cyrtodactylus (MZMU2445, MZMU2449, MZMU2542 and MZMU2545) were sequenced. Genomic DNA was extracted from ethanol (100\%) preserved liver tissue using Tissue Kit (Qiagen) following manufacturer's instructions. Sequences were generated using the primer pair MetF1 and H5934 (Macey et al. 1997). Sequence chromatograms were quality checked, edited and assembled into contigs using Sequence Scanner ver. 1.0 (Applied Biosystems). Comparative ND2 (1041 nucleotides) sequences comprising Indo-Burma clade members of Cyrtodactylus were obtained from GenBank (following Kamei \& Mahony 2021; Purkayastha et al. 2021; Table 1). Sequence alignment was done using MUSCLE (Edgar 2004) in MEGA7 (Tamura \& Nei 1993; Kumar et al. 2016) with default parameter settings. The phylogenetic reconstruction of aligned dataset was done using IQ-TREE (http://iqtree.cibiv.univie.ac.at/) online portal (Minh et al. 2020). Sequence substitution model was selected using the auto parameter with provision for FreeRate heterogeneity and the analysis was run with an ultrafast bootstrap option for 1000 iterations. Uncorrected pairwise sequence divergence was calculated in MEGA7.

\section{Abbreviations for museum collections (according to Sabaj 2020)}

$\begin{array}{ll}\text { BNHS } & =\text { Bombay Natural History Society, Mumbai, India } \\ \text { BPBM } & =\text { Bernice Pauahi Bishop Museum, Honolulu, Hawaii, USA } \\ \text { CAS } & =\text { California Academy of Sciences, San Francisco, USA } \\ \text { CES } & =\text { Centre for Ecological Sciences, Bangalore, India } \\ \text { CUMZ } & =\text { Chulalongkorn University Museum of Zoology, Bangkok, Thailand } \\ \text { FMNH } & =\text { Field Museum of Natural History, Chicago, Illinois, USA } \\ \text { MVZ } & =\text { Museum of Vertebrate Zoology, Berkeley, California, USA } \\ \text { MZMU } & =\text { Departmental Museum of Zoology, Mizoram University, Aizawl, India } \\ \text { LSUHC } & =\text { La Sierra University Herpetological Collection, La Sierra University, Riverside, }\end{array}$




\begin{tabular}{|c|c|c|}
\hline & & California, USA \\
\hline PMNH & $=$ & Pakistan Museum of Natural History, Islamabad, Islamabad Capital Territory \\
\hline VR/ERS/ZSI & $=$ & $\begin{array}{l}\text { National Zoological Collection, maintained by the North Eastern Regional } \\
\text { Centre, Zoological Survey of India, Shillong, India }\end{array}$ \\
\hline
\end{tabular}

\section{Results}

We present two distinct populations of Cyrtodactylus, one each from Umroi military cantonment area, Ri-Bhoi district, Meghalaya state and from Siaha town in the Siaha district of Mizoram state of India. Both populations are members of the $C$. khasiensis group (see Purkayastha et al. 2020; Grismer et al. 2021). The Meghalaya population was a sister of $C$. guwahatiensis from Guwahati city of Kamrup Metropolitan District, Assam state, India and the Mizoram population was a sister to recently described C. bengkhuaiai from the Aizawl District, Mizoram state, India (Fig. 2; Table 2). Based on morphology and genetic assessment, we consider both populations to be distinct species and describe them herein.

\section{Systematics}

Phylum Chordata Haeckel, 1874

Class Reptilia Laurenti, 1768

Order Squamata Oppel, 1811

Family Gekkonidae Gray, 1825

Subfamily Gekkoninae Gray, 1825

Genus Cyrtodactylus Gray, 1827

Cyrtodactylus exercitus sp. nov. urn:1sid:zoobank.org:act:820A0B94-A800-4852-BB4A-A7BDF9D1156B

Figs $1-3 \mathrm{~A}-\mathrm{C}, 4,5 \mathrm{~B}, 6$; Tables 1-3

\section{Diagnosis}

Cyrtodactylus exercitus sp. nov. is a moderate-sized gecko (adult SVL 48.2-68.0 mm); 9-11 supralabials; 9-10 infralabials; dorsal tubercles are rounded, bluntly conical and feebly keeled in 21-24 longitudinal rows; 32-34 paravertebral tubercles between the level of the axilla and the level of the groin; 35-37 midventral scale rows; $11-15$ precloacal pores in males; $16-17$ subdigital lamellae under IV toe; no single row of transversely enlarged subcaudal scales; dorsal markings are dark brown, irregular blotches with a distinctive white posterior border; tail with alternating dark and light bands.

\section{Differential diagnosis}

Genetically, Cyrtodactylus exercitus sp. nov. is a sister taxon to the lowland species C. guwahatiensis, differing from each other by an uncorrected p-distance of $4.21 \%-4.25 \%$, as in Fig. 2 and Table 2. Morphological and meristic data are shown in Table 3. Cyrtodactylus exercitus sp. nov. has been compared morphologically with all known members of the genus Cyrtodactylus within the khasiensis group. Morphologically, Cyrtodactylus exercitus sp. nov. is close to C. ayeyarwadyensis but differs from the latter by having greater TRL/SVL ratio $(0.45-0.57 .1$, avg 0.51 in C. exercitus sp. nov. and 0.40 in C. ayeyarwadyensis); in C. ayeyarwadyensis dorsum is with transverse rows of rectangular brown blotches vs W-shaped dorsal blotches in Cyrtodactylus exercitus sp. nov. Cyrtodactylus exercitus sp. nov. can be differentiated by having a smaller maximum adult size, SVL $68.0 \mathrm{~mm}$ maximum vs maximum adult size $81.7 \mathrm{~mm}$ in C. arunachalensis, $78.0 \mathrm{~mm}$ in C. ayeyarwadyensis, $77.0 \mathrm{~mm}$ in C. bapme, $72.5 \mathrm{~mm}$ in C. bengkhuaiai, $88 \mathrm{~mm}$ in C. brevidactylus, $79.78 \mathrm{~mm}$ in C. cayuensis, $96.2 \mathrm{~mm}$ in C. jaintiaensis, $80.0 \mathrm{~mm}$ in C. kazirangaensis, $81.1 \mathrm{~mm}$ in C. khasiensis, $72.0 \mathrm{~mm}$ in C. markuscombaii (Darevsky, Helfenberger, Orlov \& Shah, 1998), $82.0 \mathrm{~mm}$ in C. martinstolli, $74 \mathrm{~mm}$ in C. mombergi, $78.2 \mathrm{~mm}$ in C. montanus, 
Table 1 (continued on the next 2 pages). List of Cyrtodactylus ND2 sequences used in this study.

\begin{tabular}{|c|c|c|c|}
\hline Species & $\begin{array}{l}\text { Museum } \\
\text { No }\end{array}$ & Locality & $\begin{array}{l}\text { GenBank } \\
\text { Accession } \\
\text { No. }\end{array}$ \\
\hline Cyrtodactylus exercitus sp. nov. & MZMU2542 & $\begin{array}{l}\text { India, Meghalaya, Ri-Bhoi } \\
\text { District, Umroi }\end{array}$ & OK247679 \\
\hline Cyrtodactylus exercitus sp. nov. & MZMU2545 & $\begin{array}{l}\text { India, Meghalaya, Ri-Bhoi } \\
\text { District, Umroi }\end{array}$ & OK247680 \\
\hline Cyrtodactylus siahaensis sp. nov. & MZMU2445 & $\begin{array}{l}\text { India, Mizoram, Siaha } \\
\text { District, Siaha town }\end{array}$ & OK247677 \\
\hline Cyrtodactylus siahaensis sp. nov. & MZMU2449 & $\begin{array}{l}\text { India, Mizoram, Siaha } \\
\text { District, Siaha town }\end{array}$ & OK247678 \\
\hline $\begin{array}{l}\text { C. aaronbaueri Purkayastha, Lalremsanga, } \\
\text { Bohra, Biakzuala, Decemson, Muansanga, } \\
\text { Vabeiryureilai, Chauhan \& Rathee, 2021 }\end{array}$ & MZMU2015 & $\begin{array}{l}\text { India, Mizoram, Durtlang } \\
\text { North }\end{array}$ & MW596520 \\
\hline $\begin{array}{l}\text { C. aaronbaueri Purkayastha, Lalremsanga, } \\
\text { Bohra, Biakzuala, Decemson, Muansanga, } \\
\text { Vabeiryureilai, Chauhan \& Rathee, } 2021\end{array}$ & MZMU2020 & $\begin{array}{l}\text { India, Mizoram, Tamdil } \\
\text { National Wetland }\end{array}$ & MW596519 \\
\hline $\begin{array}{l}\text { C. agarwali Purkayastha, Lalremsanga, } \\
\text { Bohra, Biakzuala, Decemson, Muansanga, } \\
\text { Vabeiryureilai, Chauhan \& Rathee, 2021 }\end{array}$ & MZMU2158 & $\begin{array}{l}\text { India, Meghalaya, South Garo } \\
\text { Hills, Siju }\end{array}$ & MW596515 \\
\hline C. annandalei Bauer, 2003 & $\begin{array}{l}\text { CAS } \\
215722\end{array}$ & $\begin{array}{l}\text { Myanmar, Sagaing Div., } \\
\text { Alaungdaw Kathapa N.P }\end{array}$ & JX440524 \\
\hline $\begin{array}{l}\text { C. arunachalensis Mirza, Bhosale, Ansari, } \\
\text { Phansalkar, Sawant, Gowande \& Patel, } 2021\end{array}$ & CES13/1465 & $\begin{array}{l}\text { India, Arunachal Pradesh } \\
\text { State, Changlang Dist., Glow } \\
\text { Lake }\end{array}$ & MT341522 \\
\hline $\begin{array}{l}\text { C. aunglini Grismer, Wood, Thura, Win, } \\
\text { Grismer, Trueblood \& Quah, } 2018\end{array}$ & $\begin{array}{l}\text { LSUHC } \\
13948\end{array}$ & $\begin{array}{l}\text { Myanmar, Mandalay Reg., } \\
\text { Pyin Oo Lwin Dist., Kyauk } \\
\text { Nagar Cave }\end{array}$ & MH764589 \\
\hline C. ayeyarwadyensis Bauer, 2003 & $\begin{array}{l}\text { CAS } \\
212459\end{array}$ & $\begin{array}{l}\text { Myanmar, Ayeyarwady Div., } \\
\text { vicinity of Mwe Hauk Village }\end{array}$ & JX440526 \\
\hline C. battalensi Khan, 1993 & $\begin{array}{l}\text { PMNH } \\
2301\end{array}$ & $\begin{array}{l}\text { Pakistan, North-West Frontier } \\
\text { Prov., Battagram City }\end{array}$ & KC151983 \\
\hline C. bapme Kamei \& Mahony 2021 & BNHS 2756 & $\begin{array}{l}\text { India, Meghalaya State, East } \\
\text { Garo Hills Dist., Daribokgre } \\
\text { Hamlet }\end{array}$ & MW367435 \\
\hline $\begin{array}{l}\text { C. bengkhuaiai Purkayastha, Lalremsanga, } \\
\text { Bohra, Biakzuala, Decemson, Muansanga, } \\
\text { Vabeiryureilai, Chauhan \& Rathee, } 2021\end{array}$ & MZMU1985 & $\begin{array}{l}\text { India, Mizoram, Sailam } \\
\text { Community Reserved Forest }\end{array}$ & MW596516 \\
\hline $\begin{array}{l}\text { C. bengkhuaiai Purkayastha, Lalremsanga, } \\
\text { Bohra, Biakzuala, Decemson, Muansanga, } \\
\text { Vabeiryureilai, Chauhan \& Rathee, } 2021\end{array}$ & MZMU1986 & $\begin{array}{l}\text { India, Mizoram, Sailam } \\
\text { Community Reserved Forest }\end{array}$ & MW596517 \\
\hline $\begin{array}{l}\text { C. bengkhuaiai Purkayastha, Lalremsanga, } \\
\text { Bohra, Biakzuala, Decemson, Muansanga, } \\
\text { Vabeiryureilai, Chauhan \& Rathee, 2021 }\end{array}$ & MZMU1988 & $\begin{array}{l}\text { India, Mizoram, Sailam } \\
\text { Community Reserved Forest }\end{array}$ & MW596518 \\
\hline $\begin{array}{l}\text { C. bhupathyi Agarwal, Mahony, Giri, } \\
\text { Chaitanya\& Bauer, } 2018\end{array}$ & BNHS 2255 & $\begin{array}{l}\text { India, West Bengal State, } \\
\text { Kalimpong Dist., near Lower } \\
\text { Mongpong }\end{array}$ & KM255204 \\
\hline C. chamba Agarwal, Khandekar \& Bauer, 2018 & CES11/1291 & $\begin{array}{l}\text { India, Himachal Pradesh } \\
\text { State, Chamba Dist., near } \\
\text { Chamba }\end{array}$ & KM255191 \\
\hline $\begin{array}{l}\text { C. chanhomeae Bauer, Sumontha \& Pauwels, } \\
2003\end{array}$ & $\begin{array}{l}\text { CUMZ } \\
2003.62\end{array}$ & $\begin{array}{l}\text { Thailand, Saraburi Prov., } \\
\text { Phraputthabata Dist., Khun } \\
\text { Khlon Subdist., Thep Nimit } \\
\text { Cave }\end{array}$ & JX440529 \\
\hline C. chrysopylos Bauer, 2003 & $\begin{array}{l}\text { LSUHC } \\
13937\end{array}$ & $\begin{array}{l}\text { Myanmar, Shan State, } \\
\text { Taunggyi Dist., Ywnagan } \\
\text { Township }\end{array}$ & MH764604 \\
\hline
\end{tabular}


Table 1. (continued).

\begin{tabular}{|c|c|c|c|}
\hline Species & $\begin{array}{l}\text { Museum } \\
\text { No }\end{array}$ & Locality & $\begin{array}{l}\text { GenBank } \\
\text { Accession } \\
\text { No. }\end{array}$ \\
\hline C. fasciolatus (Blyth, 1861) & CES11/1337 & $\begin{array}{l}\text { India, Himachal Pradesh } \\
\text { State, Shimla Dist., near } \\
\text { Subathu }\end{array}$ & KM255184 \\
\hline C. gansi Bauer, 2003 & $\begin{array}{l}\text { CAS } \\
222412\end{array}$ & $\begin{array}{l}\text { Myanmar, Chin State, Min } \\
\text { Dat Township }\end{array}$ & JX440537 \\
\hline C. gubernatoris (Annandale, 1913) & BNHS 2207 & $\begin{array}{l}\text { India, Sikkim State, East } \\
\text { Sikkim Dist., Singtam Town }\end{array}$ & KM255181 \\
\hline $\begin{array}{l}\text { C. guwahatiensis Agarwal, Mahony, Giri, } \\
\text { Chaitanya \& Bauer, } 2018\end{array}$ & BNHS 2146 & $\begin{array}{l}\text { India, Assam State, Guwahati } \\
\text { Dist., Guwahati }\end{array}$ & KM255194 \\
\hline C. himalayanus Duda \& Sahi, 1978 & CES11/1317 & $\begin{array}{l}\text { India, Jammu and Kashmir } \\
\text { State, Kishtwar Dist., near } \\
\text { Kishtwar. }\end{array}$ & KM255187 \\
\hline $\begin{array}{l}\text { C. jaintiaensis Agarwal, Mahony, Giri, } \\
\text { Chaitanya \& Bauer, } 2018\end{array}$ & BNHS 2248 & $\begin{array}{l}\text { India, Meghalaya State, } \\
\text { Jaintia Hills Dist., near Jowai. }\end{array}$ & KM255195 \\
\hline $\begin{array}{l}\text { C. karsticola Purkayastha, Lalremsanga, } \\
\text { Bohra, Biakzuala, Decemson, Muansanga, } \\
\text { Vabeiryureilai, Chauhan \& Rathee, } 2021\end{array}$ & MZMU2153 & $\begin{array}{l}\text { India, Meghalaya, South Garo } \\
\text { Hills, Siju }\end{array}$ & MW596513 \\
\hline $\begin{array}{l}\text { C. karsticola } \text { Purkayastha, Lalremsanga, } \\
\text { Bohra, Biakzuala, Decemson, Muansanga, } \\
\text { Vabeiryureilai, Chauhan \& Rathee, 2021 }\end{array}$ & MZMU2156 & $\begin{array}{l}\text { India, Meghalaya, South Garo } \\
\text { Hills, Siju }\end{array}$ & MW596514 \\
\hline $\begin{array}{l}\text { C. kazirangaensis Agarwal, Mahony, Giri, } \\
\text { Chaitanya \& Bauer, } 2018\end{array}$ & BNHS 2147 & $\begin{array}{l}\text { India, Assam State, Golaghat } \\
\text { Dist., Kohora }\end{array}$ & KM255170 \\
\hline C. khasiensis (Jerdon, 1870) & $\begin{array}{l}\text { BNHS } \\
2249\end{array}$ & $\begin{array}{l}\text { India, Meghalaya State, } \\
\text { East Khasi Hills Dist., } \\
\text { Cherrapunjee Resort }\end{array}$ & KM255188 \\
\hline C. lawderanus (Stoliczka, 1871) & CES11/1343 & $\begin{array}{l}\text { India, Uttarakhand State, } \\
\text { Almora Dist., Almora. }\end{array}$ & KM255189 \\
\hline Cyrtodactylus lungleiensis & MZMU2428 & $\begin{array}{l}\text { India, Mizoram State, Lunglei } \\
\text { Dist., outskirts of Lunglei town }\end{array}$ & MZ645742 \\
\hline Cyrtodactylus lungleiensis & MZMU2429 & $\begin{array}{l}\text { India, Mizoram State, Lunglei } \\
\text { Dist., Ramthar Veng inside } \\
\text { Lunglei town }\end{array}$ & MZ645743 \\
\hline Cyrtodactylus lungleiensis & MZMU2432 & $\begin{array}{l}\text { India, Mizoram State, Lunglei } \\
\text { Dist., outskirts of Lunglei town }\end{array}$ & MZ645744 \\
\hline $\begin{array}{l}\text { C. meersi Grismer, Wood, Quah, Murdoch, } \\
\text { Grismer, Herr, Espinoza, Brown \& Lin, } 2018\end{array}$ & $\begin{array}{l}\text { LSUHC } \\
13455\end{array}$ & $\begin{array}{l}\text { Myanmar, Bago Reg., Yangon } \\
\text { (north) Dist., Taikkyi Township. }\end{array}$ & MH624104 \\
\hline $\begin{array}{l}\text { C. mombergi Grismer, Wood, Quah, Thura, } \\
\text { Herr \& Lin, } 2019\end{array}$ & $\begin{array}{l}\text { LSUHC } \\
14734\end{array}$ & $\begin{array}{l}\text { Myanmar, Kachin State, } \\
\text { Mohnyin Township, Indawgyi } \\
\text { W.S. }\end{array}$ & MN059875 \\
\hline $\begin{array}{l}\text { C. montanus Agarwal, Mahony, Giri, } \\
\text { Chaitanya \& Bauer, } 2018\end{array}$ & BNHS 2231 & $\begin{array}{l}\text { India, Tripura State, North } \\
\text { Tripura Dist., Phuldungsei } \\
\text { Village }\end{array}$ & KM255200 \\
\hline $\begin{array}{l}\text { C. myintkyawthurai Grismer, Wood, Quah, } \\
\text { Murdoch, Grismer, Herr, Espinoza, Brown \& } \\
\text { Lin, } 2018\end{array}$ & $\begin{array}{l}\text { CAS } \\
245200\end{array}$ & $\begin{array}{l}\text { Myanmar, Bago Reg., Central } \\
\text { Bago Yoma }\end{array}$ & MH624107 \\
\hline $\begin{array}{l}\text { C. nagalandensis Agarwal, Mahony, } \\
\text { Giri,Chaitanya \& Bauer, } 2018\end{array}$ & BNHS 2253 & $\begin{array}{l}\text { India, Nagaland State, } \\
\text { Kohima Dist., Khonoma }\end{array}$ & KM255199 \\
\hline C. novaeguineae (Schlegel, 1837) & $\begin{array}{l}\text { BPBM } \\
23316\end{array}$ & $\begin{array}{l}\text { Papua New Guinea, West } \\
\text { Sepik Prov., Parkop, Toricelli } \\
\text { Mts }\end{array}$ & JX440547 \\
\hline C. peguensis (Boulenger, 1893) & $\begin{array}{l}\text { LSUHC } \\
13454\end{array}$ & $\begin{array}{l}\text { Myanmar, Bago Reg., Myin } \\
\text { Mo Shwe Taung Pagoda }\end{array}$ & MH756190 \\
\hline C. philippinicus (Steindachner, 1867) & $\begin{array}{l}\text { FMNH } \\
236073\end{array}$ & Philippines, Romblon Island & JX440550 \\
\hline
\end{tabular}


Table 1. (continued).

\begin{tabular}{|c|c|c|c|}
\hline Species & $\begin{array}{l}\text { Museum } \\
\text { No }\end{array}$ & Locality & $\begin{array}{l}\text { GenBank } \\
\text { Accession } \\
\text { No. }\end{array}$ \\
\hline $\begin{array}{l}\text { C. pyadalinensis Grismer, Wood, Thura, Win \& } \\
\text { Quah, } 2019\end{array}$ & $\begin{array}{l}\text { LSUHC } \\
13932\end{array}$ & $\begin{array}{l}\text { Myanmar, Shan State, } \\
\text { Ywangan Township, } \\
\text { Panluang-Pyadalin Cave W.S. }\end{array}$ & MK488057 \\
\hline C. russelli Bauer, 2003 & $\begin{array}{l}\text { CAS } \\
226137\end{array}$ & $\begin{array}{l}\text { Myanmar, Sagaing Div., } \\
\text { Htamanthi W.S. }\end{array}$ & JX440555 \\
\hline $\begin{array}{l}\text { C. septentrionalis Agarwal, Mahony, Giri, } \\
\text { Chaitanya \& Bauer, } 2018\end{array}$ & BNHS 1989 & $\begin{array}{l}\text { India, Assam State, } \\
\text { Bongaigaon Dist., near } \\
\text { Abhayapuri. }\end{array}$ & MH971164 \\
\hline C. slowinskii Bauer, 2002 & $\begin{array}{l}\text { CAS } \\
210205\end{array}$ & $\begin{array}{l}\text { Myanmar, Sagaing Div.. } \\
\text { Alaungdaw Kathapa N.P. }\end{array}$ & JX440559 \\
\hline C. tibetanus (Boulenger, 1905) & $\begin{array}{l}\text { MVZ } \\
233251\end{array}$ & $\begin{array}{l}\text { China, Tibet Autonomous } \\
\text { Region, Lhasa, } 3 \mathrm{~km} \text { from of } \\
\text { Potala Palace }\end{array}$ & JX440561 \\
\hline $\begin{array}{l}\text { C. tripuraensis Agarwal, Mahony, Giri, } \\
\text { Chaitanya \& Bauer, } 2018\end{array}$ & BNHS 2238 & $\begin{array}{l}\text { India, Tripura State, Sepahijala } \\
\text { Dist., Sepahijala W.S. }\end{array}$ & KM255183 \\
\hline $\begin{array}{l}\text { C. urbanus Purkayastha, Das, Bohra, Bauer \& } \\
\text { Agarwal, } 2020\end{array}$ & $\begin{array}{l}\text { VR/ERS/ } \\
\text { ZSI/688 }\end{array}$ & $\begin{array}{l}\text { India, Assam State, Kamrup } \\
\text { Metropolitan Dist., Guwahati, } \\
\text { Basishta Temple. }\end{array}$ & MN911174 \\
\hline C. sp. Changlang1 & CES11/1349 & $\begin{array}{l}\text { India, Arunachal Pradesh } \\
\text { State, Changlang Dist., Miao }\end{array}$ & KM255179 \\
\hline C. sp. Changlang2 & CES13/1459 & $\begin{array}{l}\text { India, Arunachal Pradesh } \\
\text { State, Changlang Dist., Miao. }\end{array}$ & KM255192 \\
\hline C. sp. Khellong & CES13/1464 & $\begin{array}{l}\text { India, Arunachal Pradesh } \\
\text { State, West Kameng Dist., } \\
\text { Khellong. }\end{array}$ & KM255196 \\
\hline C. sp. Magway & $\begin{array}{l}\text { LSUHC } \\
226139\end{array}$ & $\begin{array}{l}\text { Myanmar, Magway Reg., Min } \\
\text { Bu Township, Shwesettaw W.S. }\end{array}$ & MH624118 \\
\hline
\end{tabular}

$72.0 \mathrm{~mm}$ in C. nagalandensis, $90.0 \mathrm{~mm}$ in C. tamaiensis, and $74.0 \mathrm{~mm}$ in C. urbanus. Cyrtodactylus exercitus sp. nov. has 11-15 PcP in males vs 7-8 PcP in C. aaronbaueri, 6-10 PcP in C. arunachalensis, 5-7 PcP in C. bengkhuaiai, 8-9 PcP in C. brevidactylus, 6-9 PcP in C. cayuensis, $10 \mathrm{PcP}$ in C. himalayicus, $7 \mathrm{PcP}$ in C. markuscombaii, 0-8 PcP in C. martinstolli, 8-10 PcP in C. montanus, 34-38 PcFP in C. karsticola, 26-39 PcFP in C. guwahatiensis, 29-37 PcFP in C. tripuraensis, 40 PcFP in C. tamaiensis, 16-29 PcFP in C. gansi; 21-24 rows of feebly keeled tubercles in the dorsum vs 27-30 rows in C. brevidactylus, 16-20 rows in C. chrysopylos, 19-20 rows in C. jaintiaensis, 18 rows in C. mandalayensis, 14-15 rows in C. markuscombaii,16-18 rows in C. nagalandensis and 19-21 rows in C. tripuraensis, 35-37 rows of scales between the ventrolateral folds vs 38 rows in $C$. arunachalensis, 47-49 rows in C. aunglini, 28-34 rows in C. cayuensis, 39-55 rows in C. chrysopylos, 30-35 rows in C. guwahatiensis, 33-34 rows in C. himalayicus, 40-42 rows in C. jaintiaensis, 37-43 rows in C. kazirangaensis, 32 rows in C. mandalayensis, 38-39 rows in C. markuscombaii, 57 rows in C. myaleiktaung, and 30-34 rows in C. urbanus. Cyrtodactylus exercitus sp. nov. has 16-17 subdigital lamellae beneath fourth toe vs 19-23 subdigital lamellae in C. aunglini and C. chrysopylos, 18-19 in C. brevidactylus, 10 in C. himalayicus and C. gansi, 19-22 in C. khasiensis and C. mombergi, 13 in C. martinstolli, and 18 in C. myaleiktaung. Cyrtodactylus exercitus sp. nov. has 9-11 paired dark blotches on the dorsum vs 7-8 C. gansi, 6-7 in C. kazirangaensis, 6 in C. myaleiktaung and 6-8 in C. urbanus. Cyrtodactylus exercitus sp. nov. can further be differentiated from $C$. agarwali and C. bapme by having a higher TRL/SVL ratio (min. 0.45 max. 0.57 avg. 0.51 vs min. 0.42 max. 0.48 avg. 0.45 in C. agarwali; min. 0.43 max. 0.47 avg. 0.45 in C. bapme). The presence of 32-34 rows of paravertebral tubercles between the level of axilla and groin 
separates Cyrtodactylus exercitus sp. nov. from C. aaronbaueri (36-39 rows), C. agarwali (34-38 rows), C. aunglini (36-45), C. bengkhuaiai (35-41), C. karsticola (34-39 rows), C. kazirangaensis (36-38), C. mombergi (35-42), C. montanus (37-43), C. nagalandensis (35-37) and C. septentrionalis (38-42 rows). Furthermore, Cyrtodactylus exercitus sp. nov. has small uniform scales on the underside of the tail, whereas C. cayuensis, C. khasiensis, and C. martinstolli have an enlarged median scale line.

\section{Etymology}

The specific epithet 'exercitus' is used as a noun in apposition in honour of the Indian army.

\section{Suggested common name}

Indian army's bent-toed gecko.

\section{Material examined}

\section{Holotype}

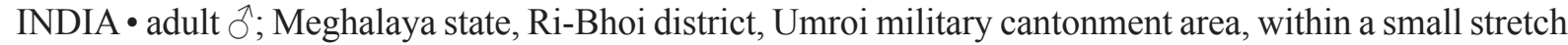
of rocky caves; $25.689753^{\circ} \mathrm{N}, 91.949835^{\circ} \mathrm{E}$; alt. $932 \mathrm{~m} ; 3$ Aug. 2021; Y. S. Rathee and J. Purkayastha leg.; rock substrata (Fig. 7A); GenBank accession number OK247679; MZMU2542 (Figs. 3A, 4, 5A).

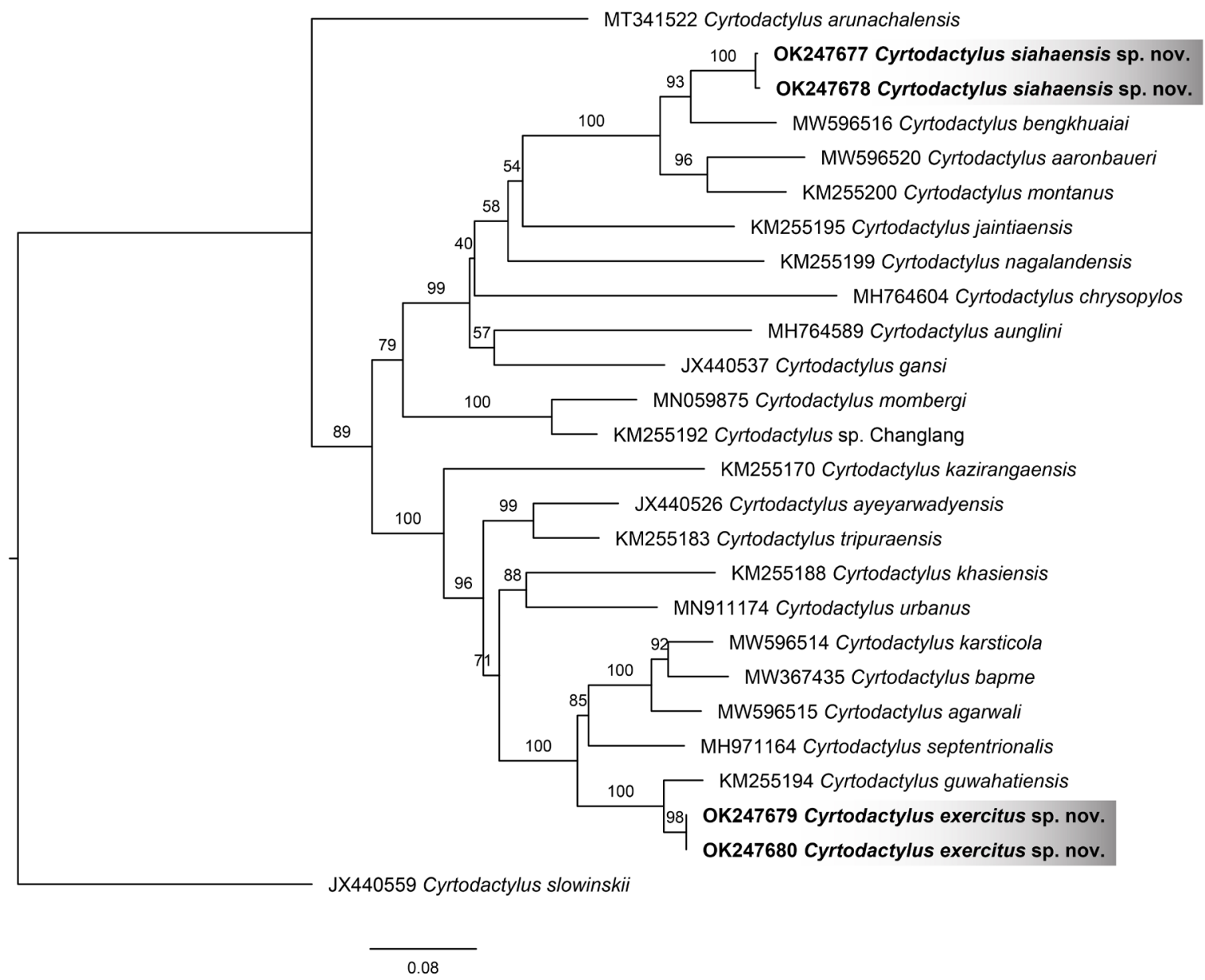

Fig. 2. Maximum Likelihood phylogeny of the Cyrtodactylus khasiensis group; numbers at nodes represent bootstrap support (preceding the species name is the NCBI accession number). 


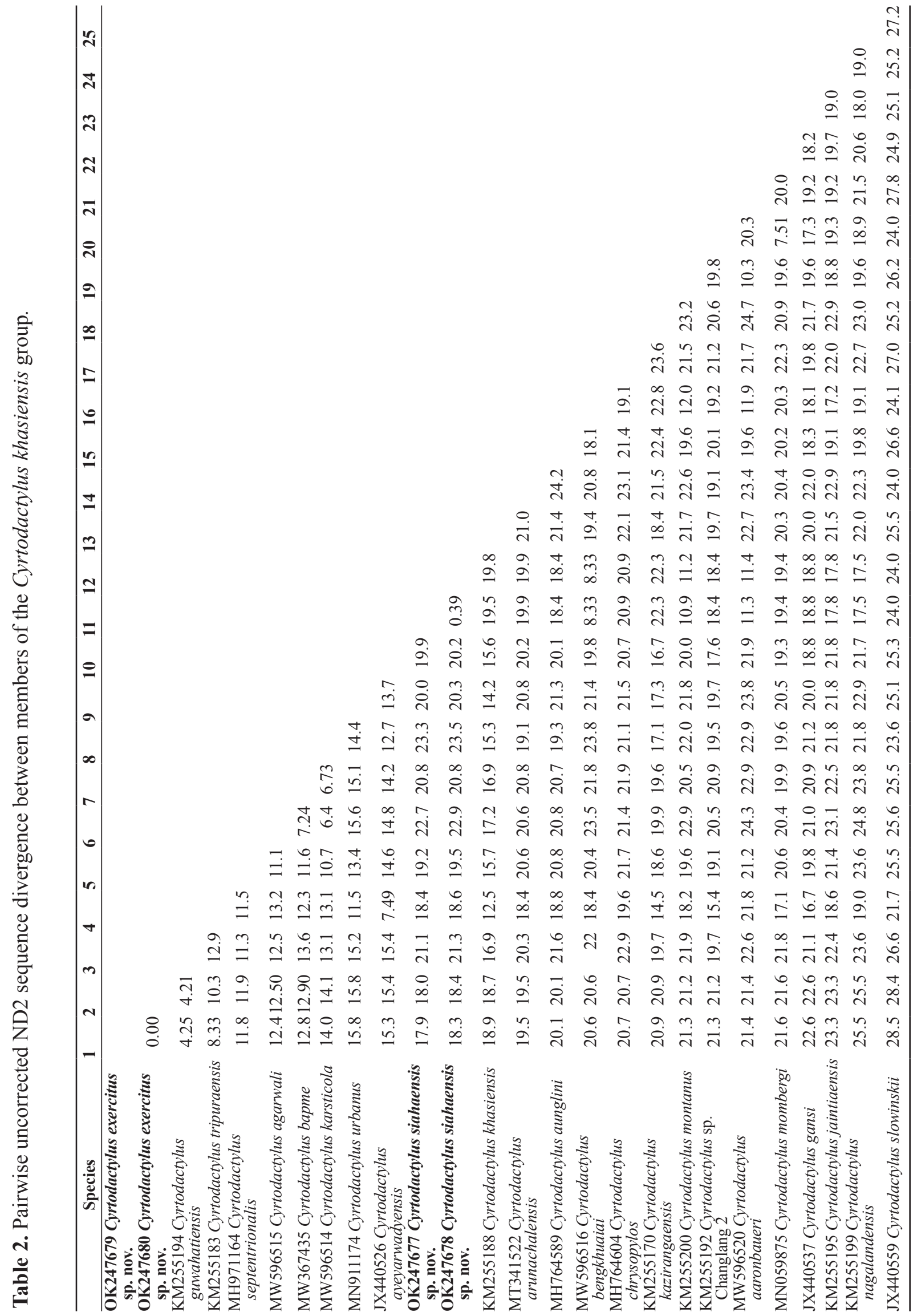


Paratypes $(n=3)$

INDIA • adult $q$; same collection data as for holotype; MZMU2543 (Figs 3B, 6), • adult ổ; same collection data as for holotype; MZMU2544 (Fig. 6) • adult $\widehat{\delta}$; same collection data as for holotype; GenBank accession number OK247680; MZMU2545 (Figs 3C, 6).

\section{Description of holotype}

Holotype is in generally good preservation condition (Fig. 4). Adult male, SVL $68.0 \mathrm{~mm}$.

HEAD. Head length slightly more than one-quarter of the snout to vent length (HL/SVL 0.29), longer than broad (HW/HL 0.63), somewhat depressed (HD/HW 0.59), and distinct from the neck; loreal region with granular scales; interorbital region is concave; canthus rostralis broadly rounded; snout is less than half of the head length (SO/HL 0.39), less than twice as long as the orbit diameter (OD/SO 0.56); scales on the forehead, canthus rostralis snout homogeneous. Scales from the posterior margin of the eyes to the nape are smaller than those of the forehead, somewhat blunt and juxtaposed; scales on forehead till the interorbital region without distinct tubercles. Orbit diameter is less than one-quarter of the head length (OD/HL 0.22) (Fig. 4D); pupil vertical with crenulate margins; superciliary scales are decreasing in size towards posterior of the eye; ear opening small (EL/HL 0.12) oval, obliquely orientated; eye to ear distance is shorter than the eye diameter (OE/OD 0.96). Rostrum slightly wider than long (RL/RW 0.89), partially divided dorsally by a weakly developed rostral groove; single enlarged supranasal on either side, separated by a single scale, little larger than enlarged scales on the snout; rostrum in contact with the first supralabials, nasals, supranasals and internasals; nostrils round, opening laterally orientated, three-fourth covered by the nasal pad, each nasal in broad contact with the rostral and surrounded by a supranasal, first supralabial, and three postnasals (Fig. 4E); mental wider than long (ML/MW 0.81), triangular; two well developed postmentals on either side, the inner pair less than twice the size of the outer pair (PMIIL/ PMIL 0.8); inner postmentals in contact with the mental, infralabial I, one outer postmental and two gular scales; outer postmental on each side is in contact with one inner postmental, infralabials I and II, and two to four gular scales; ten and eleven supralabials on each right and left side respectively, nine and ten infralabials on each right and left side respectively, infralabials II to IV bordered ventrally by a row of

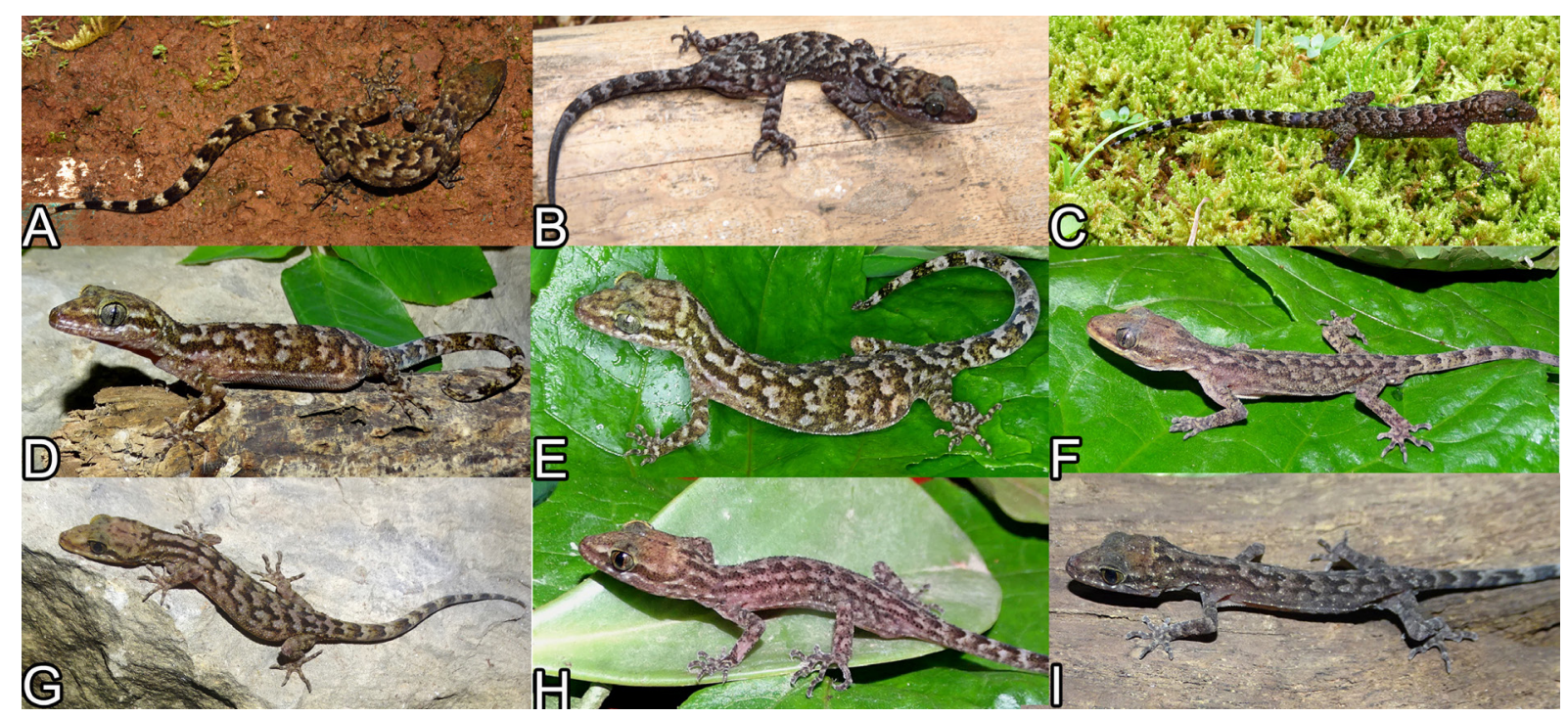

Fig. 3. Cyrtodactylus exercitus sp. nov. in life (A. MZMU2542. B. MZMU2543. C. MZMU2545); Cyrtodactylus siahaensis sp. nov. in life(D. MZMU2443. E. MZMU2444. F. MZMU2445. G. MZMU2446. H. MZMU2449. I. uncollected). 


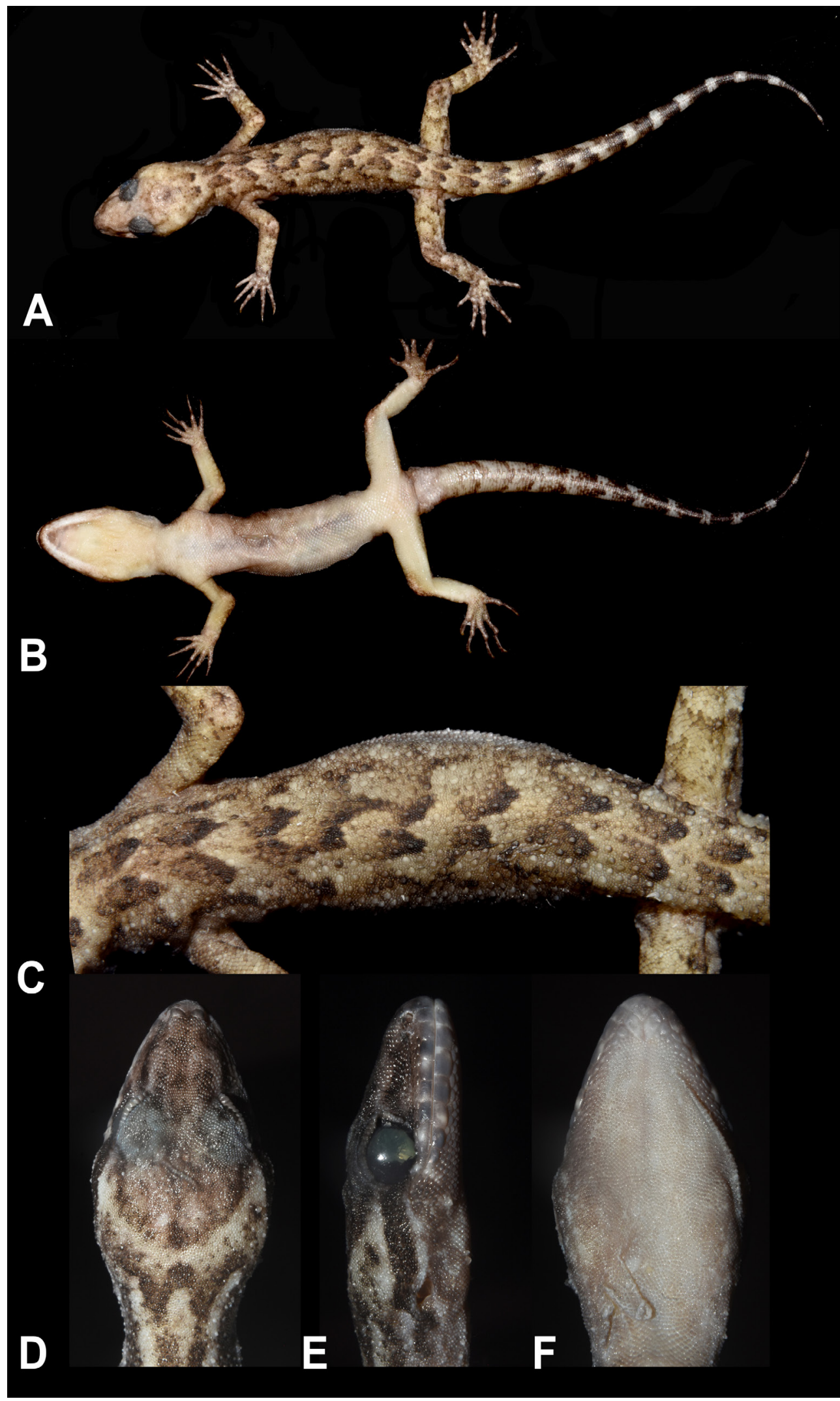

Fig. 4. Cyrtodactylus exercitus sp. nov., holotype (MZMU2542). A. Dorsal view. B.Ventral view. C. Details of dorsal pholidosis. D. Dorsal view of head. E. Lateral view of head. F. Ventral view of head. 
Table 3. (continued on the next page). Morphometric and meristic data for Cyrtodactylus exercitus sp. nov. and $C$. siahaensis sp. nov. (all in millimeters).

\begin{tabular}{|c|c|c|c|c|c|c|c|c|c|c|}
\hline \multirow{3}{*}{$\begin{array}{l}\text { Species } \\
\text { Voucher } \\
\text { no. } \\
\text { Locality }\end{array}$} & \multicolumn{4}{|c|}{ Cyrtodactylus exercitus sp. nov. } & \multicolumn{6}{|c|}{ Cyrtodactylus siahaensis sp. nov. } \\
\hline & $\begin{array}{l}\text { MZMU2542 } \\
\text { (holotype) }\end{array}$ & MZMU2543 & MZMU2544 & MZMU2545 & $\begin{array}{l}\text { MZMU2445 } \\
\text { (holotype) }\end{array}$ & MZMU2443 & MZMU2444 & MZMU2446 & MZMU2448 & MZMU2449 \\
\hline & $\begin{array}{l}\text { Umroi, } \\
\text { Meghalaya }\end{array}$ & $\begin{array}{l}\text { Umroi, } \\
\text { Meghalaya }\end{array}$ & $\begin{array}{l}\text { Umroi, } \\
\text { Meghalaya }\end{array}$ & $\begin{array}{l}\text { Umroi, } \\
\text { Meghalaya }\end{array}$ & $\begin{array}{l}\text { Siaha, } \\
\text { Mizoram }\end{array}$ & $\begin{array}{l}\text { Siaha, } \\
\text { Mizoram }\end{array}$ & $\begin{array}{l}\text { Siaha, } \\
\text { Mizoram }\end{array}$ & $\begin{array}{l}\text { Siaha, } \\
\text { Mizoram }\end{array}$ & $\begin{array}{l}\text { Siaha, } \\
\text { Mizoram }\end{array}$ & $\begin{array}{l}\text { Siaha, } \\
\text { Mizoram }\end{array}$ \\
\hline Sex & $\mathrm{M}$ & $\mathrm{F}$ & $\mathrm{M}$ & $\mathrm{M}$ & M & $\mathrm{F}$ & $\mathrm{F}$ & M & $\mathrm{F}$ & $\mathrm{F}$ \\
\hline SVL & 68 & 66.78 & 48.16 & 61.8 & 57.38 & 63.96 & 72 & 61.14 & 42.62 & 43.24 \\
\hline TRL & 36 & 31 & 27.5 & 27.52 & 29 & 32.92 & 33.9 & 30 & 22 & 22.3 \\
\hline BW & 9.5 & 9.88 & 6.78 & 7.84 & 8.18 & 11.44 & 11.5 & 9.7 & 5.4 & 5.5 \\
\hline TL & 81.12 & - & 58 & 68.64 & 65.4 & 69.72 & 72.58 & - & 52.8 & 52 \\
\hline TW & 5.42 & 4.5 & 3.8 & 4.64 & 4.02 & 4.34 & 5.48 & 5 & 3.36 & 3.36 \\
\hline HL & 19.4 & 19.62 & 14.24 & 18.14 & 18.36 & 19.38 & 21.02 & 19.22 & 19 & 19.1 \\
\hline HW & 12.28 & 12.12 & 8.98 & 10.84 & 10.18 & 10.54 & 12.4 & 10.32 & 7.48 & 7.52 \\
\hline HD & 7.26 & 6.52 & 5.06 & 6.28 & 6.24 & 6.3 & 7.5 & 6.24 & 4.82 & 4.78 \\
\hline FL & 10.58 & 10.2 & 8.2 & 9.8 & 9.6 & 9.82 & 10.64 & 9.2 & 7.8 & 7.98 \\
\hline CL & 12.24 & 12.3 & 9.28 & 11.42 & 10.94 & 11.16 & 12.4 & 11 & 8.6 & 8.58 \\
\hline OD & 4.24 & 4.22 & 3.3 & 4.28 & 4 & 3.8 & 4.2 & 3.82 & 3 & 3.1 \\
\hline NO & 4.52 & 4.6 & 3 & 3.9 & 4 & 4.44 & 5.2 & 4.5 & 2.7 & 2.72 \\
\hline SO & 7.52 & 6 & 5.18 & 6.42 & 5.08 & 6.82 & 7.8 & 6.92 & 4.82 & 4.86 \\
\hline $\mathrm{OE}$ & 4.08 & 4.54 & 2.6 & 3.34 & 3.32 & 4.04 & 4.4 & 4.12 & 2.24 & 2.28 \\
\hline EL & 2.24 & 2 & 1.56 & 2 & 1.58 & 1.7 & 1.94 & 1.5 & 1.42 & 1.4 \\
\hline IN & 1.7 & 2 & 1.2 & 2.06 & 1.56 & 1.48 & 1.94 & 1.54 & 0.8 & 0.82 \\
\hline IO & 3 & 3 & 2.1 & 2.64 & 2.32 & 2.56 & 2.92 & 2.54 & 1.72 & 1.72 \\
\hline $\mathrm{F} 1$ & 4.82 & 5 & 3.94 & 4.6 & 4.4 & 4.12 & 5 & 4.42 & 3.72 & 3.76 \\
\hline F2 & 6.2 & 6.2 & 4.92 & 6.5 & 5.42 & 5 & 5.4 & 5.66 & 4.24 & 4.28 \\
\hline F3 & 6.64 & 6.6 & 5.6 & 6.8 & 5.66 & 5.36 & 5.7 & 5.72 & 4.32 & 4.36 \\
\hline F4 & 7 & 7.8 & 6.52 & 7.8 & 6.8 & 6.4 & 7.26 & 6.08 & 4.46 & 4.5 \\
\hline F5 & 6.74 & 7.6 & 5.52 & 6.38 & 5.9 & 5.4 & 5.86 & 5.8 & 4.36 & 4.4 \\
\hline $\mathrm{T} 1$ & 6.06 & 5.8 & 4.6 & 5.4 & 4.82 & 4.84 & 4.94 & 4.96 & 4.2 & 4.4 \\
\hline $\mathrm{T} 2$ & 7.4 & 6.9 & 5.74 & 7.34 & 6.2 & 5.22 & 6.24 & 6 & 4.36 & 4.38 \\
\hline $\mathrm{T} 3$ & 8.12 & 8.1 & 6.6 & 8 & 6.9 & 6.32 & 7.6 & 6.9 & 5 & 5 \\
\hline $\mathrm{T} 4$ & 8.4 & 8.48 & 7.1 & 8.78 & 7.44 & 7.8 & 8.16 & 7.6 & 5.72 & 5.8 \\
\hline T5 & 8 & 8.53 & 6.3 & 8.2 & 7 & 6.74 & 7.8 & 6.6 & 5.16 & 5.24 \\
\hline RL & 2 & 2 & 1.2 & 2.1 & 1.52 & 1.7 & 1.76 & 1.64 & 1.18 & 1.2 \\
\hline RW & 2.24 & 2.2 & 1.8 & 2.24 & 1.68 & 2 & 2 & 1.92 & 1.24 & 1.26 \\
\hline $\begin{array}{l}\mathrm{PcP} / \\
\mathrm{PcFP}\end{array}$ & 15 & - & 12 & 11 & 7 & - & - & 7 & - & - \\
\hline MVSR & 37 & 37 & 35 & 35 & 34 & 36 & 35 & 37 & 34 & 37 \\
\hline DTR & 22 & 21 & 22 & 24 & 23 & 24 & 22 & 22 & 22 & 24 \\
\hline PVT & 32 & 34 & 32 & 34 & 36 & 38 & 38 & 36 & 39 & 39 \\
\hline SL(L/R) & 10.11 & 10.9 & 11.11 & 10.10 & 10.9 & 10.10 & 10.11 & 10.10 & 10.10 & 10.11 \\
\hline IL(L/R) & 9.10 & 10.10 & 9.9 & 9.9 & 9.9 & 8.10 & 11.10 & 10.9 & 10.10 & 10.9 \\
\hline
\end{tabular}


Table 3. (continued).

\begin{tabular}{|c|c|c|c|c|c|c|c|c|c|c|}
\hline \multirow{3}{*}{$\begin{array}{l}\text { Species } \\
\text { Voucher } \\
\text { no. } \\
\text { Locality }\end{array}$} & \multicolumn{4}{|c|}{ Cyrtodactylus exercitus sp. nov. } & \multicolumn{6}{|c|}{ Cyrtodactylus siahaensis sp. nov. } \\
\hline & $\begin{array}{l}\text { MZMU2542 } \\
\text { (holotype) }\end{array}$ & MZMU2543 & MZMU2544 & MZMU2545 & $\begin{array}{l}\text { MZMU2445 } \\
\text { (holotype) }\end{array}$ & 5 MZMU2443 & MZMU2444 & MZMU2446 & MZMU2448 & MZMU2449 \\
\hline & $\begin{array}{l}\text { Umroi, } \\
\text { Meghalaya }\end{array}$ & $\begin{array}{l}\text { Umroi, } \\
\text { Meghalaya }\end{array}$ & $\begin{array}{l}\text { Umroi, } \\
\text { Meghalaya }\end{array}$ & $\begin{array}{l}\text { Umroi, } \\
\text { Meghalaya }\end{array}$ & $\begin{array}{l}\text { Siaha, } \\
\text { Mizoram }\end{array}$ & $\begin{array}{l}\text { Siaha, } \\
\text { Mizoram }\end{array}$ & $\begin{array}{l}\text { Siaha, } \\
\text { Mizoram }\end{array}$ & $\begin{array}{l}\text { Siaha, } \\
\text { Mizoram }\end{array}$ & $\begin{array}{l}\text { Siaha, } \\
\text { Mizoram }\end{array}$ & $\begin{array}{l}\text { Siaha, } \\
\text { Mizoram }\end{array}$ \\
\hline Sex & $\mathrm{M}$ & $\mathrm{F}$ & M & M & M & $\mathrm{F}$ & $\mathrm{F}$ & $\mathrm{M}$ & $\mathrm{F}$ & $\mathrm{F}$ \\
\hline$\overline{\mathrm{F} 1 \mathrm{~L}}$ & $4(2) 6$ & $4(1) 6$ & $4(1) 6$ & $4(1) 8$ & $4(1) 6$ & $3(1) 6$ & $5(3) 5$ & $4(1) 6$ & $4(1) 6$ & $4(1) 7$ \\
\hline F2L & $5(1) 8$ & $6(2) 6$ & $4(0) 8$ & $4(2) 8$ & $5(1) 7$ & $5(1) 7$ & $6(1) 8$ & $5(2) 7$ & $5(1) 7$ & $4(1) 8$ \\
\hline F3L & $5(1) 10$ & $6(0) 10$ & $5(0) 9$ & $5(3) 8$ & $5(1) 8$ & $5(2) 8$ & $5(2) 9$ & $5(2) 9$ & $5(1) 8$ & $4(0) 10$ \\
\hline F4L & $6(1) 10$ & $6(1) 9$ & $5(0) 10$ & $5(2) 9$ & $5(1) 8$ & $5(1) 8$ & $6(2) 8$ & $5(1) 8$ & $6(2) 8$ & $5(0) 8$ \\
\hline F5L & $4(3) 8$ & $5(0) 8$ & $3(1) 8$ & $6(3) 7$ & $3(1) 7$ & $4(1) 8$ & $4(0) 8$ & $5(1) 8$ & $5(1) 7$ & $4(1) 9$ \\
\hline $\mathrm{T} 1 \mathrm{~L}$ & $5(0) 7$ & $4(1) 8$ & $4(1) 7$ & $4(3) 7$ & $3(1) 7$ & $3(1) 7$ & $3(2) 7$ & $3(2) 6$ & $5(2) 6$ & $4(2) 7$ \\
\hline $\mathrm{T} 2 \mathrm{~L}$ & $5(1) 9$ & $6(0) 9$ & $5(1) 9$ & $6(1) 9$ & $4(1) 8$ & $5(1) 9$ & $5(1) 8$ & $5(1) 8$ & $6(1) 8$ & $5(1) 9$ \\
\hline T3L & $6(1) 10$ & $6(1) 10$ & $6(1) 10$ & $5(4) 9$ & $5(0) 11$ & $6(3) 8$ & $5(1) 10$ & $6(0) 10$ & $6(1) 8$ & $5(1) 9$ \\
\hline T4L & $6(3) 10$ & $7(1) 10$ & $7(1) 10$ & $7(1) 10$ & $7(0) 11$ & $7(3) 8$ & $6(2) 9$ & $7(1) 11$ & $7(1) 11$ & $7(1) 10$ \\
\hline T5L & $5(1) 9$ & $6(1) 10$ & $4(1) 8$ & $6(2) 11$ & $5(2) 9$ & $6(2) 8$ & $7(3) 9$ & $5(1) 10$ & $6(2) 9$ & $5(1) 10$ \\
\hline $\begin{array}{l}\text { Dorsal } \\
\text { spots }\end{array}$ & 9 & 10 & 11 & 9 & 9 & 8 & 9 & 8 & 9 & 10 \\
\hline
\end{tabular}

enlarged gular scales, largest anteriorly; gular region mostly covered with small granular scales except for a few rows bordering the mental, postmentals and infralabials which are larger, flat and juxtaposed (Fig. 4F).

BoDy. Moderately slender; trunk length is half of the snout to vent length (TRL/SVL 0.53); dorsal scales heterogeneous, mostly rounded granular scales, intermixed with irregularly arranged, enlarged tubercles, bluntly conical and keeled throughout, becoming more keeled, conical and slightly smaller towards dorsolateral side and the flanks; largest on the pre sacral and sacral regions; tubercles extend posteriorly from the occipital region to beyond the tail base; tubercles on the nape are smaller than those on the dorsum; 22 mid-body rows of dorsal tubercles; 32 paravertebral tubercles between the level of the axilla and the level of the groin (Fig. 4C); ventral scales are larger than dorsals, smooth, cycloid, imbricate to subimbricate, smallest in the throat region gradually becoming enlarged, with largest being in the mid-abdominal region. Thirty-seven mid-ventral scale rows (Fig. 4B); fifteen precloacal pores in a continuous series with six pitted scales on the femoral region on the left and right sides interrupted by seven and six non pitted or pored scales, respectively (Fig. 5A). Scales posteriorly bordering the porebearing scale series are almost of the same size as the pore-bearing scales; three post cloacal tubercles on each side of the tail base.

LIMBS. Forearm (FL/SVL 0.16) and tibia (CL/SVL 0.18) short; digits laterally compressed, without a scansorial pad, strongly inflected at each joint, all bearing robust, recurved claws; subdigital lamellae transversely widened beneath the basal phalanx; basal lamellae 4-5-5-6-4 on the left manus, 5-5-6-6-5 on the left pes; distal lamellae (intervening rows of nonlamellar granular scales between basal and distal lamellae series in parentheses): 6(2)-8(1)-10(1)-10(1)-8(3) on the left manus, 7(0)-9(1)-10(1)-10(3)$9(1)$ on the left pes; interdigital webbing absent from both manus and pes; relative length of digits: $\mathrm{I}<$ II $<$ III $<$ V $<$ IV on the left manus, I $<$ II $<$ V $<$ III $<$ IV on the left pes; scales on the palms and soles are smooth, weakly raised, subimbricate; scales on the forelimbs are heterogeneous in size, comprising 
flat, subimbricate scales, ventral portion covered with heterogenous sized imbricate scales; scales on the hindlimbs are heterogeneous in size, dorsal surfaces of thighs and shanks with small granular scales, intermixed with scattered, enlarged, conical, keeled tubercles; strongly keeled tubercle on the tibia region; ventral surfaces of hindlimbs with enlarged, smooth, imbricate scales.
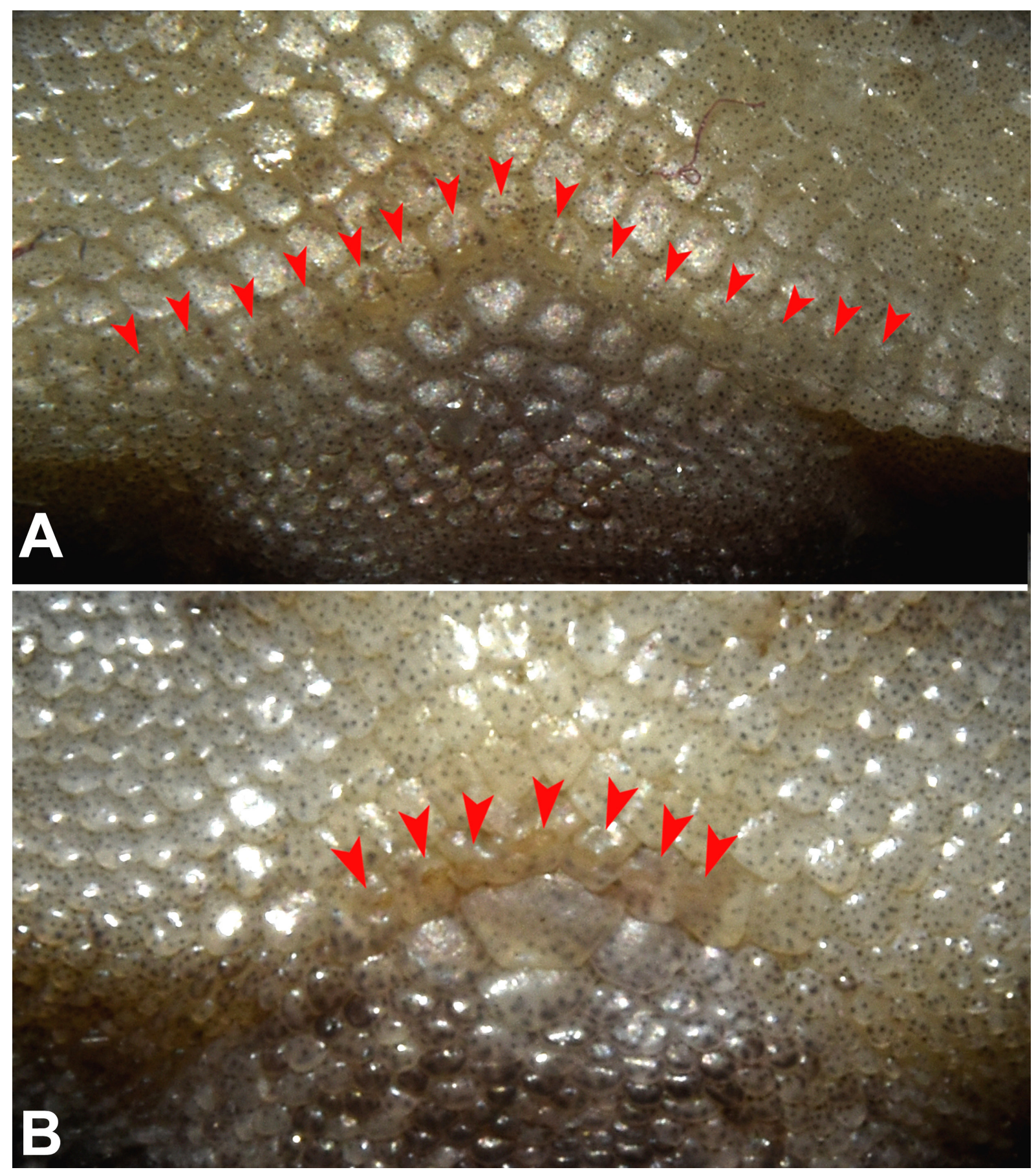

Fig. 5. The arrangement of precloacal pores in A. Cyrtodactylus exercitus sp. nov., holotype (MZMU2542) B. Cyrtodactylus siahaensis sp. nov (MZMU2445). 
TAIL. Original, entire, gradually tapering from the base to the tip; dorsal caudal scales smooth, rounded, subimbricate, large at anterior region becoming smaller posteriorly; poorly developed tubercles limited to the anterior part of the tail. Ventral caudal scales much enlarged than the dorsal caudal scales; no transversely enlarged subcaudal plates.

Colouration IN Life. Dorsum of body grayish brown; head is primarily brown in colour with a few yellowish patches in the snout region and gray patches towards the posterior end of the head; supra ocular brownish yellow in colour. Nape has a white "W" streak with two dark brown blotches in it. Lateral surface of the post ocular region without any streak. The dorsal surface with nine pairs of dark brown vertebral blotches, each forming a "W" shaped pattern and have a distinct white border at the posterior surface of the dark brown "W" patterns (Fig. 3A). The ventral region is off-white in colour.

The tail has alternating light (13) and dark (14) bands. The first three dark bands are "W" shaped and the light bands are dirty white in colour.

Colouration In PRESERVATIVE. The colour is pale in comparison to the live specimen. The dark spots on the dorsum have turned brownish-black (Fig. 4).

\section{Taxonomic remarks}

Refer to Table no. 3 for meristic, morphometric and basic pholidosis variation within the type series of Cyrtodactylus exercitus sp. nov. The type series of $C$. exercitus sp. nov. is comprised of three males (two

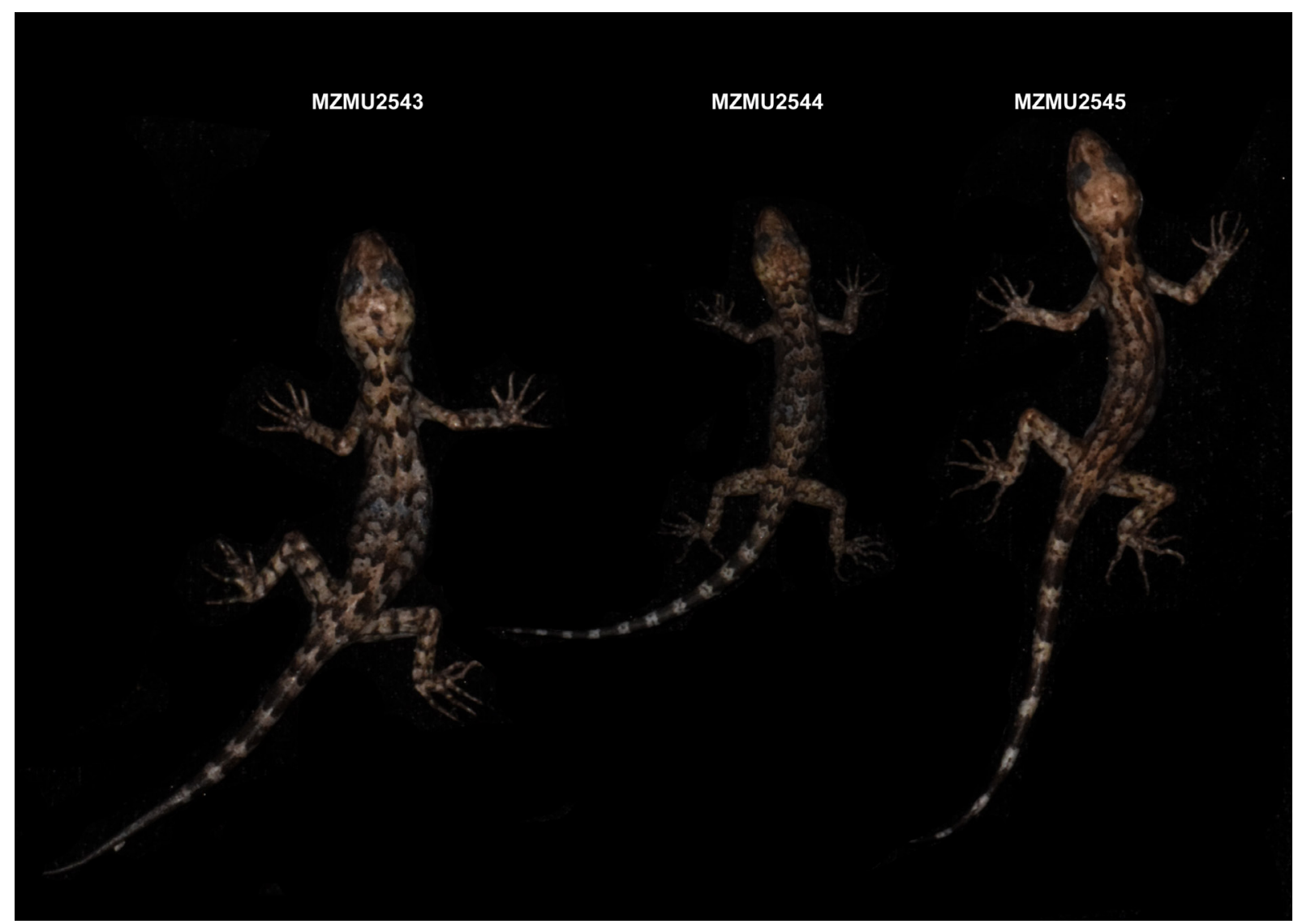

Fig. 6. Type series of Cyrtodactylus exercitus sp. nov. 
adults (MZMU2542, MZMU2545) and a sub-adult (MZMU2544), and one female (an adult MZMU2543). Paratype MZMU2543 has visibly pitted scales (11) in its precloacal region.

\section{Ecology and distribution}

This species is only known from the type locality inside the Umroi army cantonment area. The type series was collected from a very small rocky patch within the cantonment area (Fig. 7A). All the individuals were collected just after dark from the crevices of the rock. During our survey, we could not find any Cyrtodactylus within the whole cantonment area except in this patch.

Cyrtodactylus siahaensis sp. nov. urn:1sid:zoobank.org:act:CC5D593B-6611-4C72-BEAB-144F8595D61C

Figs 3D-I, 5B, 8, 9; Tables 1-3

\section{Diagnosis}

Cyrtodactylus siahaensis sp. nov. is a moderate-sized gecko (adult SVL 42.6-63.9 mm); 9-11 supralabials; 9-11 infralabials; dorsal tubercles are rounded, bluntly conical and feebly keeled in 22-24 longitudinal
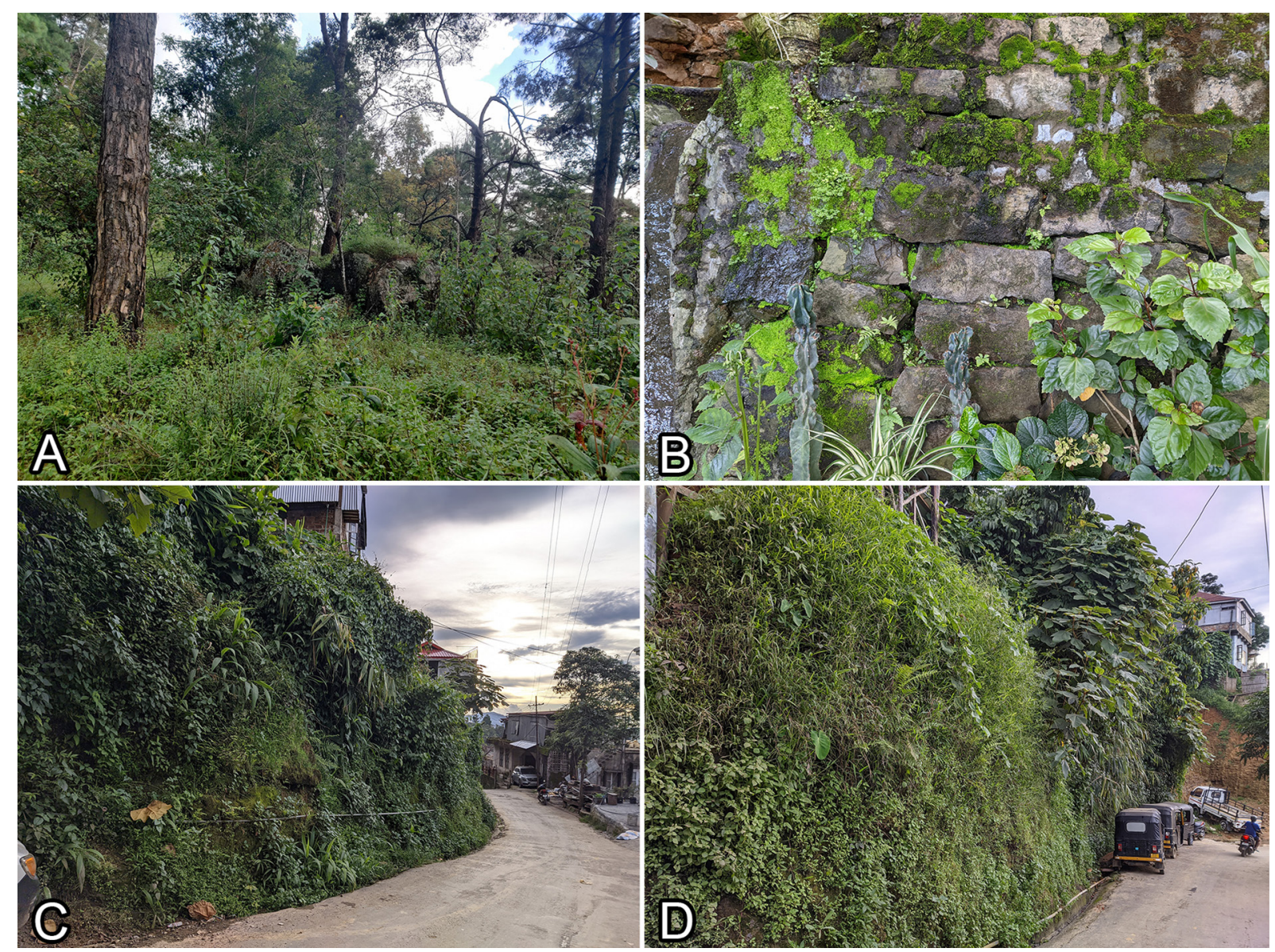

Fig. 7. Microhabitats showing the collection sites of our newly described species of Cyrtodactylus from Northeast India. A. Collection site of Cyrtodactylus exercitus sp. nov. in Umroi military cantonment area, Ri-Bhoi district, Meghalaya state. B-D. Collection sites of C. siahaensis sp. nov. in Siaha town, Siaha district, Mizoram state. 


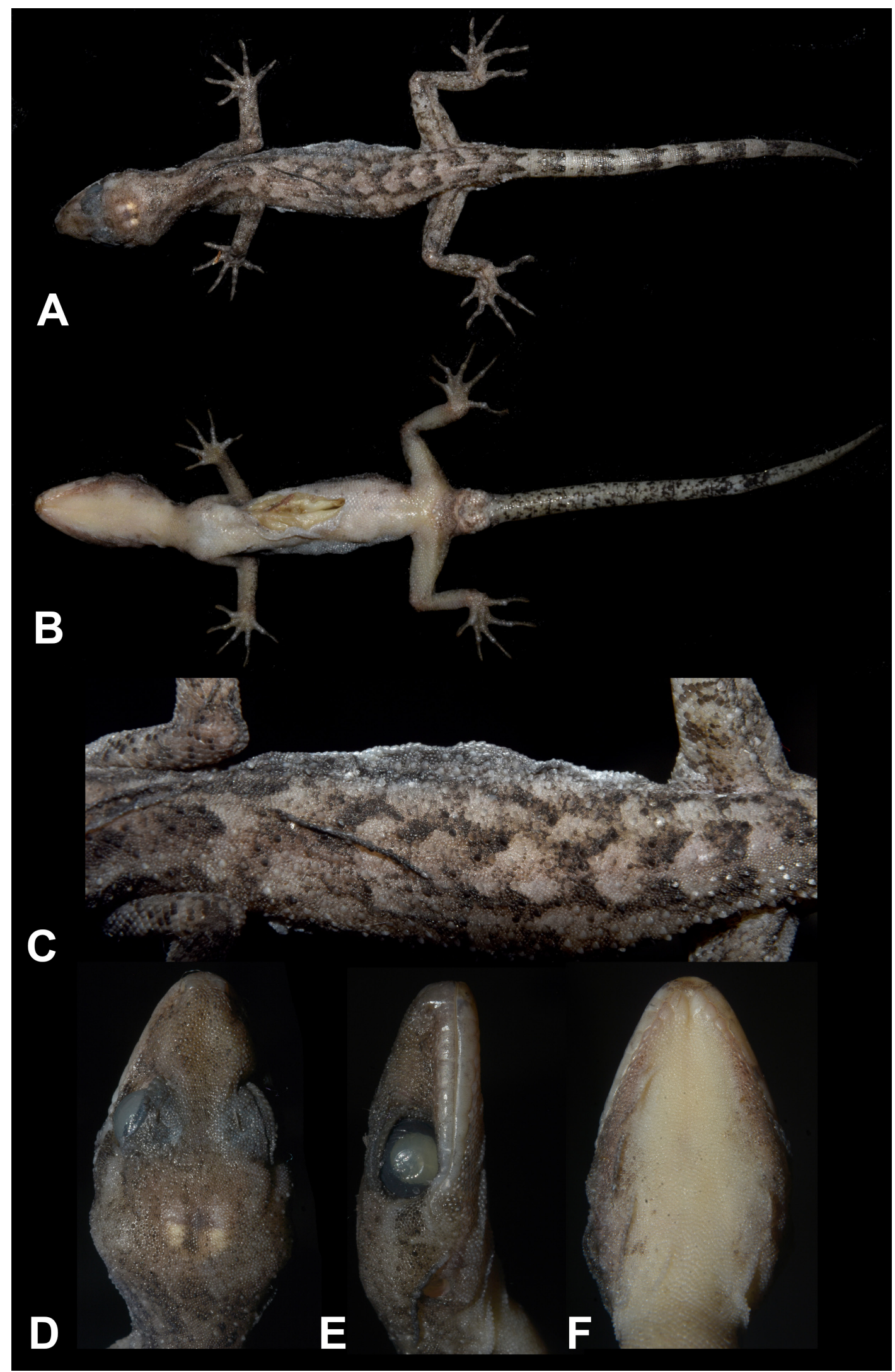

Fig. 8. Cyrtodactylus siahaensis sp. nov., holotype (MZMU2445). A. Dorsal view. B. Ventral view. C. Details of dorsal pholidosis. D. Dorsal view of head. E. Lateral view of head. F. Ventral view of head. 
rows; 36-39 paravertebral tubercles between the level of the axilla and the level of the groin; 34-37 mid-ventral scale rows; 7 precloacal pores in males; 15-18 subdigital lamellae under IV toe; no single row of transversely enlarged subcaudal scales; dorsal markings are dark brown, irregular blotches; tail with alternating dark and light bands.

\section{Differential diagnosis}

Genetically, Cyrtodactylus siahaensis sp. nov. is a sister to C. bengkhuaiai differing from each other by an uncorrected p-distance of $8.33 \%$ as in Fig. 2 and Table 2. Morphological and meristic measurements are as shown in Table 3. Cyrtodactylus siahaensis sp. nov. has been compared morphologically with all known members of the genus Cyrtodactylus within the khasiensis group. Morphologically, Cyrtodactylus siahaensis sp. nov. can be differentiated from $C$. exercitus sp. nov. by having a larger maximum adult size, $72.0 \mathrm{~mm}$ max. (vs $68.0 \mathrm{~mm}$ max. in Cyrtodactylus exercitus sp. nov.), seven precloacal pores in a continuous series vs 11-15 precloacal pores in C. exercitus and 36-39 paravertebral tubercles between the level of axilla and groin vs 32-34 tubercles in C. exercitus.

Cyrtodactylus siahaensis sp. nov. differs from other members of the $C$. khasiensis group by the following characters: a moderate body size with a maximum SVL of $72.0 \mathrm{~mm}$ max. vs $81.7 \mathrm{~mm}$ in C. arunachalensis, $78 \mathrm{~mm}$. in C. ayeyarwadyensis, $81.6 \mathrm{~mm}$ in C. aunglini, $77.0 \mathrm{~mm}$ in C. bapme, $88.0 \mathrm{~mm}$ in C. brevidactylus, $83.8 \mathrm{~mm}$ in C. chrysopylos, $62.3 \mathrm{~mm}$ in C. gansi, $64.5 \mathrm{~mm}$ in C. himalayicus, $96.2 \mathrm{~mm}$ in C. jaintiaensis, $80 \mathrm{~mm}$. in C. kazirangaensis, $81.1 \mathrm{~mm}$ in C. khasiensis, $61.7 \mathrm{~mm}$ in $C$. mandalayensis, $82 \mathrm{~mm}$ in C. martinstolli, $78.2 \mathrm{~mm}$ in C. montanus, $59.0 \mathrm{~mm}$ in C. myaleiktaung and $90 \mathrm{~mm}$ in C. tamaiensis; $7 \mathrm{PcP}$

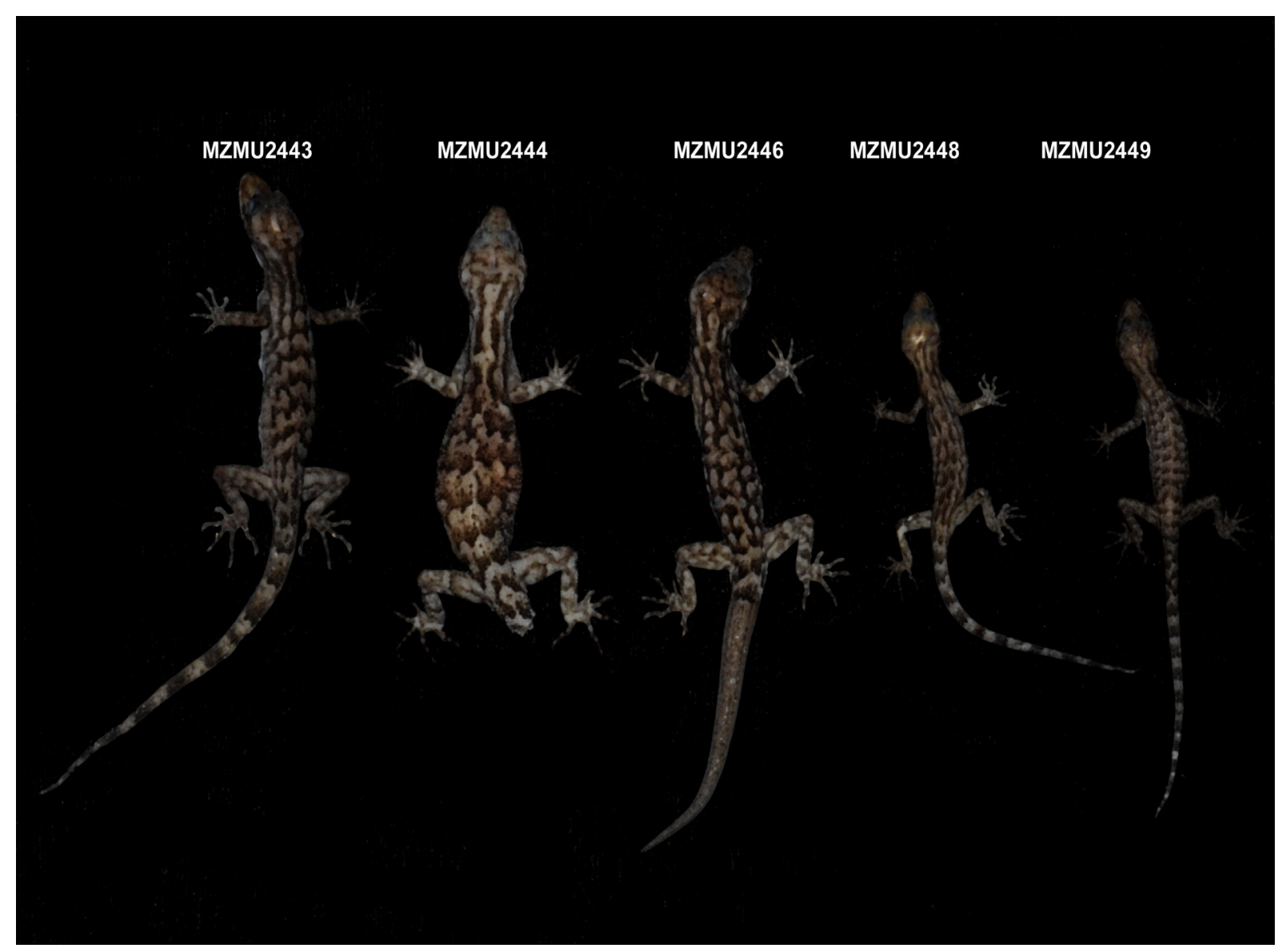

Fig. 9. Type series of Cyrtodactylus siahaensis sp. nov. 
in a continuous series in males vs 10-28 PcP in C. ayeyarwadyensis, 11-18 PcP in C. agarwali, 12-13 $\mathrm{PcP}$ in C. aunglini, 5-7 PcP in C. bengkhuaiai, 8 or $9 \mathrm{PcP}$ in C. brevidactylus, 8-13 PcP in C. chrysopylos, 10 in C. himalayicus, 11-12 PcP in C. jaintiaensis, 10-11 PcP in C. kazirangaensis, 10-12 PcP in C. khasiensis, 0-8 $\mathrm{PcP}$ in C.martinstolli, 9-11 $\mathrm{PcP}$ in C. mombergi, 8-10 PcP in C. montanus, $14 \mathrm{PcP}$ in C. septentrionalis, 9-12 PcP in C. urbanus, 16-29 PcFP in C. gansi, 26-39 PcFP in C. guwahatiensis, 34-38 PcFP in C. karsticola, 40 PcFP in C. tamaiensis, 29-37 PcFP in C. tripuraensis, ; 22-24 rows of tubercles throughout the dorsum vs 24-26 in C. arunachalensis, 27-30 rows in C. brevidactylus, 16-20 rows in C.chrysopylos, 19-21 rows in C. himalayicus, 19-20 rows in C. jaintiaensis, 18 rows in C. cayuensis and C. mandalayensis, 14-15 rows in C. markuscombaii, 16-18 rows in C. nagalandensis, 21 rows in C. tamaiensis, 19-21 rows in C. tripuraensis, 34-37 rows of scales across the venter vs 38 rows in C. arunachalensis, 47-49 rows in C. aunglini, 37-42 rows in C. bengkhuaiai, 28-34 rows in C. cayuensis, 39-55 rows in $C$. chrysopylos, 40-42 rows in C. jaintiaensis, 37-43 rows in C. kazirangaensis, 32 rows in C. mandalayensis, 38-39 rows in C. markuscombaii, 57 rows in C. myaleiktaung and 30-34 in C. urbanus; 36-39 paravertebral tubercles between the level of axilla and groin vs 30-35 in C. chrysopylos and 30-34 in C. jaintiaensis; 15-18 subdigital lamellae beneath toe IV vs 19-22 subdigital lamellae in C. khasiensis and C. mombergi; 13 in C. martinstolii and 10 in C. gansi; 8-10 dark blotches in the dorsum vs 11-12 blotches in C. aaronbaueri, six in C. myaleiktaung; indistinct light and dark blotches in C. karsticola and C. nagalandensis and an indistinct pattern of thick dark reticulations enclosing lighter blotches in C.montanus; a higher TRL/SVL ratio ( $\min .0 .47$ max. 0.52 avg.0.5 vs min. 0.42 max. 0.48 avg. 0.45 in C.agarwali, min. 0.35 max. 0.46 avg. 0.43 in C.guwahatiensis, min. 0.44 max. 0.47 avg. 0.46 in C.septentrionalis, min 0.43, max 0.49, avg 0.46 in C.bengkhuaiai, min. 0.43 max. 0.47 avg. 0.45 in C.bapme). Cyrtodactylus siahaensis sp. nov. can again be differentiated from C. aaronbaueri by having a lower TRL/SVL ratio, min. 0.47 max. 0.52 avg. 0.5 vs $\min 0.5$, max 0.56 , avg 0.52 in C. aaronbaueri. Cyrtodactylus siahaensis sp. nov. also differs from C.bapme by having 0-3 pitted scales in females (vs 0-13 precloacal pits in the latter). Furthermore, Cyrtodactylus siahaensis sp. nov. can again be differentiated from $C$. ayeyarwadyensis by the absence of white punctuations bordering the dark dorsal spots in the dorsum (vs presence of dark blotches usually bordered posteriorly by white punctuations in C. ayeyarwadyensis). In Cyrtodactylus siahaensis sp. nov. the underneath of the tail with small uniform scale vs enlarged median scale line in C. cayuensis, C. khasiensis, C. martinstolli.

\section{Etymology}

The origin of specific epithet 'siahaensis' is derived from the name of the town, Siaha (a district capital of Siaha District) from where the type series was collected.

\section{Suggested common name}

Siaha bent-toed gecko.

\section{Suggested local name}

Khotlia (Mara ethnic language, which means bent-toed geckos).

\section{Holotype}

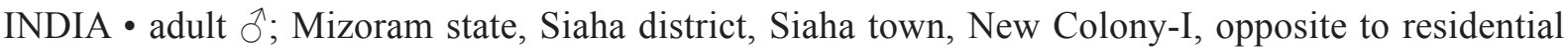
buildings nearby Meisavaih road; $22.490565^{\circ} \mathrm{N}, 92.9789525^{\circ} \mathrm{E} ; 1025 \mathrm{~m}$ a.s.l.; 13 Jul. 2021; B. Litho, B. Nohro, V. Mathipi, L. Biakzuala and H.T. Lalremsanga leg.; retaining wall (Fig. 7B); GenBank accession number OK247677; MZMU2445 (Figs 3F, 8).

\section{Paratypes $(n=5)$}

INDIA $\bullet 1$ adult ${ }^{\top}$; same collection data as for holotype; MZMU2444 (Figs 3E, 9) 1 adult $\odot$; Mizoram state, Siaha district, Siaha town, New Colony-I, roadside rocky wall near Evangelical Church of Maraland; 
22.490098 ${ }^{\circ}$, $92.980885^{\circ}$ E; elevation 1055 m a.s.1.; 13 Jul. 2021; B. Litho, B. Nohro and L. Muansanga leg.; crevices and horizontal cleft (Fig. 7C); MZMU2443 (Figs 3D, 9) 1 adult ${ }^{\lambda}$; same collection data as for preceding; MZMU2446 (Figs 3G, 9) • 1 juvenile $\odot$; same collection data as for preceding; MZMU2448 (Fig. 9) - 1 juvenile +; Mizoram state, Siaha district, Siaha town, New Colony-I; $22.489605^{\circ} \mathrm{N}$, 92.978440 ${ }^{\circ}$ E; elevation $1038 \mathrm{~m}$ a.s.1.; 13 Jul. 2021; B. Litho, B. Nohro and H.T. Lalremsanga leg.; rock bedding wall (Fig. 7D); GenBank accession number OK247678; MZMU2449 (Figs 3H, 9).

\section{Description of holotype}

Holotype is in generally good preservation condition with a crease on the nape region (Fig. 8A-F). Adult male, SVL $57.4 \mathrm{~mm}$.

HEAD. Longer than broad (HW/HL 0.55), about one-third of SVL (HL/SVL 0.32), distinct from neck and somewhat depressed (HD/HW 0.61) interorbital area flat, canthus rostralis broadly rounded; loreal region inflated with granular scales; snout less than half of the head length (SO/HL 0.28 ); scales on the canthus rostralis, snout and forehead are homogeneous; scales from the posterior margin of the eyes to the nape are slightly smaller than those of the forehead, somewhat blunt and juxtaposed; scales on the interorbital and occipital regions somewhat heterogeneous in size, having indistinct tubercles; eye approximately one-quarter of the head length (OD/HL 0.22) (Fig. 8D); vertical pupil with crenulate margins; supraciliaries moderate in size, blunt and somewhat oval and circular, those present in the midportion of the supraciliaries (above the orbit) are large and more prominent; ear opening oval, obliquely orientated, small (EL/HL 0.09); orbit to ear distance is less than the eye diameter (OE/OD 0.83); rostrum wider than long (RL/RW 0.90), partially and dorsally divided by a poorly developed rostral groove; single enlarged supranasal on either side, separated by two small granular internasals which are about the same size as enlarged scales on the snout; rostral in contact with the first supralabials, nasals, two supranasals and two internasals; nostrils semicircular, openings laterally orientated, posterior half covered by the nasal pad, each nasal is in broad contact with the rostral and surrounded by the supranasal, first supralabial, and three postnasals (Fig. 8E); a single row of small granular scales separate the orbit from the supralabials; mental wider than long (ML/MW 0.65), triangular; two well developed postmentals on either side; the inner pair of postmentals more than one and a half of the size of the outer pair (PMIIL/PMIL 0.6), bordered by the mental, infralabial I, the outer postmental and four gular scales; outer postmentals bordered by inner postmental, infralabials I and II, and four to five gular scales on either side; ten supralabials on the left and nine on the right side, bordered by a row of medium to large sized scales, somewhat flat and elongated; nine infralabials on each side, a row of enlarged gular scales (largest anteriorly) ventrally bordering infralabials I to III; gular region with small granular scales throughout except for a few scale rows bordering the mental, postmentals and infralabials which are larger, flat and juxtaposed (Fig. 8F).

BoDy. Moderately slender, trunk length approximately half of the snout to vent length (TRL/SVL 0.51); dorsal scales are mostly heterogeneous, small rounded granule like scales intermixed with irregularly arranged, bluntly conical and feebly keeled enlarged tubercles (3-4 times the size of granular scales) which becomes more conical and slightly smaller towards the flanks, the largest on the sacral region; tubercles extending posteriorly from the occipital region to the second segment of the tail and are smaller on the nape than those of the dorsum; 23 mid-dorsal tubercle rows; 36 paravertebral tubercles between the level of the axilla and the level of the groin (Fig. 8C); ventrolateral folds are poorly developed, not denticulate but with a single row of continuous, projected smooth tubercles; ventral scales smooth, cycloid (Fig. 8B), imbricate to subimbricate, much larger than dorsals and are slightly smaller in size under thighs; 34 midventral scale rows; seven distinct precloacal pores in a continuous series (Fig. 5B); a single row of three scales (median scale being the largest) between the precloacal pores and the vent; a weakly developed postcloacal tubercle on each side of the tail base. 
LimBs. Forearm (FL/SVL 0.17) and tibia (CL/SVL 0.19) short; digits narrow, without a scansorial pad, strongly inflected at each joint, all bearing robust, recurved claws; sub-digital lamellae transversely widened beneath the basal phalanx; basal lamellae 4-5-5-5-3 on the right manus, 3-4 -5-7-5 on the right pes; distal lamellae (intervening rows of nonlamellar granular scales between the basal and distal lamellae series in parentheses): $6(1)-7(1)-8(1)-8(1)-7(1)$ on the right manus, $7(1)-8(1)-11(0)-11(0)$ $-9(2)$ on the right pes; interdigital webbing absent from both manus and pes; relative length of digits: I $<$ II $<$ III $<$ V $<$ IV on the right manus, I $<$ II $<$ III $<$ V $<$ IV on the right pes; scales on the palms and soles are smooth, weakly raised, subimbricate; scales on the forelimbs are heterogeneous in size, comprising flat,imbricate to subimbricate scales on the upper arms, and those on forearms are heterogeneous in size, ventral portion covered with heterogenous sized imbricate scales; scales on the hindlimbs are heterogeneous in size, dorsal surfaces of the thighs and shanks have slightly larger scales, intermixed with scattered, enlarged, conical, feebly keeled tubercles; anterior portion of thighs and ventral aspect of hindlimbs have enlarged, smooth, imbricate scales.

TAIL. Regenerated from the seventh tail segment, oval in cross-section, dorsoventrally depressed; dorsal tubercles on the tail base (tail portion containing the hemipenes) are enlarged, flat, and pointed; few enlarged, flat and distinctly keeled, tubercles randomly scattered near the first segment of the tail, remaining dorsal caudal scales smooth, flat, heterogenous in size and shape; subcaudal scales smooth, imbricate and heterogeneous in size, forming a mid-ventral series of granular scales; no enlarged transverse or paired row of subcaudals.

COLOURATION IN LIFE. Dorsum brown in colour with a greenish tinge; head is primarily greenish brown; nape has a indistinct 'W-shaped' marking intermixed with whitish spots; an indistinct dark brown coloured postocular stripe present which runs till the anterior margin of ear opening. Eight pairs of dark brown blotches present on the dorsum, somewhat forming a reticulated pattern; hind limbs and forelimbs have indistinct whitish or cream coloured blotches intermixed with light brown blotches. Tail (regenerated from the seventh segment) has alternating dark and light bands (Fig. 3F). The ventral region is off-white in colour.

COLOURATION IN PRESERVATIVE. The colour is pale in comparison to the live specimen. The dark spots on the dorsum have darkened to brownish-black (Fig. 8).

\section{Taxonomic remarks}

Refer to Table 3 for meristic, morphometric and basic pholidosis variation within the type series of Cyrtodactylus siahaensis sp. nov. The type series of $C$. siahaensis sp. nov. comprises two adult males (MZMU 2445 and MZMU 2446) and four females (two adults (MZMU2443 and MZMU2444) and two sub-adults (MZMU2448 and MZMU2449)). Paratype MZMU2444 has three visible pitted scales on its precloacal region.

\section{Ecology and distribution}

This species is only known from the type locality near human settlements in the centre of Siaha town (Fig. 7B-D). The type series was collected from an area within $1 \mathrm{~km} 2$ on 13 July 2021 from inside and outside of crevices and clefts of rock bedding. Being a strictly nocturnal species, they are most active two to five hours after dark and are mostly seen in and around crevices, clefts, rock beddings and rock walls. All the individuals were encountered and collected from a height of approximately $90 \mathrm{~cm}$ to 170 $\mathrm{cm}$ above the ground. All handled specimens were very active, offensive and inclined to bite. The habitat is comprised largely of anthropogenic area with small patches of homestead garden under moderately disturbed secondary growth tropical evergreen forest. All the collection sites are located in the vicinity of water sources, like small hill stream, spring and well with rocky moist, damp and shady places. Vegetation around microhabitats include, Ageratum conyzoides (L.) L., Calocasia sp., Chromolaena odorata (L.) 
R.M.King \& H.Rob., Cynodon dactylon (L.) Pers., Hibiscus sp., Mikania micrantha Kunth, Mimosa pudica L., Molineria crassifolia Baker, Semecarpus anacardium L.f., Thysanolaena latifolia (Roxb. ex Hornem.) Honda, Viburnum sp. etc. Most surface areas of the rocky microhabitat were covered by patches of lichens and bryophytes, while more shady areas were flourished with ferns and grasses.

\section{Discussion}

Northeast India is a part of Eastern Himalayas and Indo-Burma biodiversity hotspots. The region is a confluence zone of Indo-Chinese, Indo-Malayan and Indian biogeographic elements (Ahmed et al. 2009). Recently Northeast India has also shown the presence of Sri Lankan elements (Agarwal et al. 2021; Lalremsanga et al. 2021). Being located in the southernmost part of the Northeastern India, Mizoram and Meghalaya are part of the north-western extremity of the Indo-Burma biodiversity hotspot. Given its high diversity of landforms and climatic zones, Indo-Burma harbours a relatively large array of habitats and biodiversity. Indo-Burma hotspot's geological and evolutionary history contributes to a high level of endemism and diversity (Tordoff et al. 2020). Historically, the region was home to just a single species, C. khasiensis; the other 13 species have been discovered since 2018. With detailed evaluation of the C. khasiensis group (see Agarwal 2018a) more species have been uncovered, most of which are morphologically similar.

The state of Meghalaya is home to five species of Cyrtodactylus, Cyrtodactylus exercitus sp. nov. being the sixth member of the group. Agarwal et al. (2018b) divided C. khasiensis group into lowland and highland clades, based on the altitudinal distribution and genetic make-up of the species. For all the species so far described from Northeast India, the clade structure of Agarwal et al. (2018b) is recovered. However, $C$. exercitus sp. nov. is seen as an exception, in that this species is from a highland locality (932 m a.s.1.), but belongs genetically to a lowland clade, with C. guwahatiensis being its sister species.

Recent studies in Mizoram have resulted in the discovery of new reptilian species (e.g., Vogel et al. 2017, 2020; Giri et al. 2019a, 2019b ; Lalronunga et al. 2021; Purkayastha et al. 2021) and distributional range extensions (e.g., Lalremsanga et al. 2010, 2011, 2018a, 2018b; Ashaharraza et al. 2019; Lalbiakzuala \& Lalremsanga 2019a, 2019b; Hmar et al. 2020, 2021a; 2021b; Muansanga et al. 2020). Until this study, only four species of Bent-toed geckos, C. khasiensis (Mathew 2007), C. montanus (Muansanga et al. 2020), C. aaronbaueri and C. bengkhuaiai were recorded from Mizoram state with C. khasiensis being provisionally removed from the state fauna of Mizoram (Purkayastha et al. 2021). All the known species of Cyrtodactylus from the state are represented by the highland clade so far, and another member in this clade $C$. siahaensis sp. nov. is recovered from the present survey, whose distribution is limited to the Siaha town area in the southern part of Mizoram, while C. bengkhuaiai is confined to the hill arrays of Sailam in the central part of the state, and the other two species, C. aaronbaueri and C. montanus, are found in the northern part of the state. All the individuals collected in different habitats throughout the Siaha town belonged to $C$. siahaensis.

Siaha is the third largest town after the state capital, Aizawl, and Lunglei, it is located $140 \mathrm{~km}$ south of Aizawl in aerial distance. In winter, the temperature varies from $8^{\circ} \mathrm{C}$ to $21^{\circ} \mathrm{C}$ while in summer it ranges between $26^{\circ} \mathrm{C}$ and $35^{\circ} \mathrm{C}$. The vegetation of the area falls under the tropical semi-evergreen forest associated with moist deciduous forest, corresponding to the semi-evergreen 2B/C2 forest type (Champion \& Seth 1968 ) and the average annual rainfall of Siaha District is $2564.5 \mathrm{~mm}$ (Guhathakurta et al. 2020). There is scanty information on the herpetofauna of southern part of Mizoram, except Pawar and Birand (2001).

The new gecko species are both nocturnal and are rarely encountered. Very little is known about their natural history. We suggest that the new species described here should also be considered Data Deficient (DD) according to the categorisation of IUCN (2022), due to the fact that all the specimens were known 
only from this area. Our knowledge on many reptile species of Northeast India is limited and detail further research should be done here.

\section{Acknowledgements}

We are grateful to the Chief Wildlife Warden, Environment, Forests and Climate Change Department, Government of Meghalaya and Mizoram for the herpetofaunal collection permit within Meghalaya state (Permit no. FWC/G/173/Pt-V/2377-87) and Mizoram state (Permit no. A.33011/2/99-CWLW/225). We acknowledge the following funding agencies: Department of Biotechnology (DBT, New Delhi), sanction no. DBT-NER/AAB/64/2017; Defence Research and Development Organization (DRDO, New Delhi), sanction no. DFTM/07/3600/NESTC/EWM/M/P-01/01; National Mission for Himalayan Studies (NMHS, Uttarakhand), sanction no. GBPNI/NMHS-2017/MG-22. We are also thankful to Byhnahmosa Nohro, Ht. Decemson, Malsawmdawngliana Fanai, Gospel Zothanmawia Hmar, Lal Rinsanga and Roma Hmar for their assistance in fieldwork and laboratory.

\section{References}

Agarwal I., Bauer A.M., Jackman T.R. \& Karanth K.P. 2014 Insights into Himalayan biogeography from geckos: A molecular phylogeny of Cyrtodactylus (Squamata: Gekkonidae). Molecular Phylogenetics and Evolution 80: 145-155. https://doi.org/10.1016/j.ympev.2014.07.018

Agarwal I., Mahony S., Giri V.B., Chaitanya R. \& Bauer A.M. 2018a. Two new species of Cyrtodactylus Gray, 1827 (Squamata: Gekkonidae) with comments on name-bearing types from Northeast India. Zootaxa 4420 (3): 334-356. https://doi.org/10.11646/zootaxa.4420.3.2

Agarwal I., Mahony S., Giri V.B., Chaitanya R. \& Bauer A.M. 2018b. Six new Cyrtodactylus (Squamata: Gekkonidae) from Northeast India. Zootaxa 4524 (5): 501-535. https://doi.org/10.11646/zootaxa.4524.5.1

Agarwal I., Kamei R.G. \& Mahony S. 2021. The phylogenetic position of the enigmatic Assam day gecko Cnemaspis cf. assamensis (Squamata: Gekkonidae) demonstrates a novel biogeographic connection between Northeast India and south India-Sri Lanka, Amphibia-Reptilia 42 (3): 355-367.

https://doi.org/10.1163/15685381-bja10062

Ahmed M.F., Das A. \& Dutta S.K. 2009. Amphibians and Reptiles of Northeast India: A Photographic Guide. Aaranyak, Guwahati.

Ashaharraza K., Rangasamy V., Lalremsanga H.T., Lalbiakzuala, Sailo J. \& Charlton T. 2019. A new state record of the Mandarin Rat Snake Euprepiophis mandarinus (Cantor, 1842) (Squamata: Colubridae: Coronellini) from Mizoram, India. Amphibian and Reptile Conservation 13 (1): 230-234.

Bauer A.M. 2003 Descriptions of seven new Cyrtodactylus (Squamata: Gekkonidae) with a key to the species of Myanmar (Burma). Proceedings of the California Academy of Sciences 54: 463-498.

Champion S.H.G. \& Seth S.K. 1968. A Revised Survey of the Forest Types of India. The Manager of Publicaton, Govt. of India, New Delhi.

Darevsky I.S., Helfenberger N., Orlov N. \& Shah K. 1998. Two new species of the genus Gonydactylus (Sauria: Gekkonidae) from eastern Nepal. Russian Journal of Herpetology 4 (2): 89-93.

https://doi.org/10.30906/1026-2296-1997-4-2-89-93

Edgar R.C. 2004. MUSCLE: multiple sequence alignment with high accuracy and high throughput. Nucleic Acids Research 32 (5): 1792-1797. https://doi.org/10.1093/nar/gkh340

Giri V.B., Chaitanya R., Mahony S., Lalronunga S., Lalrinchhana C., Das A., Sarkar V., Karanth P. \& Deepak V. 2019a. On the systematic status of the genus Oriocalotes Günther, 1864 (Squamata: Agamidae: 
Draconinae) with the description of a new species from Mizoram state, Northeast India. Zootaxa 4638 (4): 451-484. https://doi.org/10.11646/zootaxa.4638.4.1

Giri V.B., Gower D.J., Das A., Lalremsanga H.T., Lalronunga S., Captain A. \& Deepak V. 2019b. A new genus and species of natricine snake from Northeast India. Zootaxa 4603 (2): 241-264.

https://doi.org/10.11646/zootaxa.4603.2.2

Grismer L.L., Wood Jr. P.L., Poyarkov N.A., Le M.D., Kraus F., Agarwal I., Oliver P.M., Nguyen S.N., Nguyen T.Q., Karunarathna S., Welton L.J., Stuart B.L., Luu V.Q., Bauer A.M., O'Connell K.A., Quah E.S.H., Chan K.O., Ziegler T., Ngo H., Nazarov R.A., Aowphol A., Chomdej S., Suwannapoom C., Siler C.D., Anuar S., Tri N.V. \& Grismer J.L. 2021. Phylogenetic partitioning of the third-largest vertebrate genus in the world, Cyrtodactylus Gray, 1827 (Reptilia; Squamata; Gekkonidae) and its relevance to taxonomy and conservation. Vertebrate Zoology 71: 101-154. https://doi.org/10.3897/vz.71.e59307

Guhathakurta P., Bandgar A., Menon P., Prasad A.K., Sangwan N. \& Advani S.C. 2020. Observed Rainfall Variability and Changes Over Mizoram State. Met Monograph No.: ESSO/IMD/HS/Rainfall Variability/18(2020)/42. India Meteorological Department. Ministry of Earth Sciences. Government of India.

Hmar G.Z., Lalbiakzuala, Lalmuansanga, Zote D., Vanlalhruaia Ramengmawii H.B., Das K.C. \& Lalremsanga H.T. 2020. A first distribution record of the Indian Peacock Softshell Turtle Nilssonia hurum (Gray, 1830) (Reptilia: Testudines: Trionychidae) from Mizoram, India. Journal of Threatened Taxa 12 (14): 17036-17040. https://doi.org/10.11609/jott.6712.12.14.17036-17040

Hmar G.Z., Lalbiakzuala, Lalmuansanga, Zote D., Vanlalhruaia Ramengmawii H.B., Decemson Ht., Das K.C. \& Lalremsanga H.T. 2021a. First record of the exotic Red-eared Slider, Trachemys scripta elegans (Wied 1838) (Emydidae), from Mizoram, India. Reptiles \& Amphibians 28 (1): 52-53.

https://doi.org/10.17161/randa.v28i1.15310

Hmar G.Z., Ramengmawii H.B., Biakzuala L., Zote D., Hruaia V., Liana T., Lalremsanga H.T. \& Das K.C. 2021b. New records of the endangered Southeast Asian Box Turtle, Cuora amboinensis (Testudines, Geoemydidae), from Mizoram, Northeast India. Herpetology Notes 14: 1117-1180.

IUCN. 2022. Guidelines for Using the IUCN Red List Categories and Criteria. Version 15. Available from https://nc.iucnredlist.org/redlist/content/attachment_files/RedListGuidelines.pdf [accessed on 2 Feb. 2022].

Kamei R.G. \& Mahony S. 2021. A new species of Bent-toed gecko (Squamata: Gekkonidae: Cyrtodactylus Gray, 1827) from the Garo Hills, Meghalaya State, north-east India, and discussion of morphological variation for C. urbanus. Herpetological Journal 31 (3): 177-196. https://doi.org/10.33256/31.3.177196

Kumar S., Stecher G. \& Tamura K. 2016. MEGA7: molecular evolutionary genetics analysis version 7.0 for bigger datasets. Molecular Biology and Evolution 33 (7): 1870-1874.

https://doi.org/10.1093/molbev/msw054

Lalbiakzuala \& Lalremsanga H.T. 2019a. Geographic distribution notes: Hebius venningi. Herpetological Review 50 (2): 330.

Lalbiakzuala \& Lalremsanga H.T. 2019b. Geographic distribution notes: Lycodon fasciatus. Herpetological Review 48 (1): 129.

Lalremsanga H.T., Khawlhring L. \& Lalrotluanga 2010. Three additional lizard (Squamata: Sauria) records for Mizoram, India. Journal of Threatened Taxa 2 (2): 718-720.

https://doi.org/10.11609/JoTT.o2246.718-20

Lalremsanga H.T., Sailo S. \& Chinliansiama H. 2011. Diversity of snakes (Reptilia: Squamata) and role of environmental factors in their distribution in Mizoram, Northeast India. In: Diwakari (ed.) Proceedings of Advances in Environmental Chemistry 64: 265-269. Excel Indian Publishers, New Delhi. 
Lalremsanga H.T., Lalrinsanga \& Lalbiakzuala. 2018a. Geographic Distribution Notes: Protobothrops mucrosquamatus. Herpetological Review 48 (1): 131.

Lalremsanga H.T., Lalrinsanga, Vanlalchhuana M., Vanlalhrima \& Vogel G. 2018b. First Record of the Species Gongylosoma scriptum (Theobald, 1868) (Squamata: Colubridae) From India. Hamadryad 38 (1 \& 2): 12-19.

Lalronunga S., Lalhmangaiha K., Zosangliana I., Lalhmingliani E., Gower D. J., Das A. \& Deepak V. 2021. A new species of Stoliczkia Jerdon, 1870 (Serpentes: Xenodermidae) from Mizoram, India. Zootaxa 4996 (3): 555-568. https://doi.org/10.11646/zootaxa.4996.3.9

Li P.P. 2007. Description of a new subspecies of Cyrtodactylus khasiensis from China. Acta Zootaxonomica Sinica 32: 733-737.

Macey J.R., Larson A., Ananjeva N.B., Fang Z. \& Papenfuss T.J. 1997. Two novel gene orders and the role of light-strand replication in rearrangement of the vertebrate mitochondrial genome. Molecular Biology and Evolution 14: 91-104. https://doi.org/10.1093/oxfordjournals.molbev.a025706

Mahony S. 2009. Taxonomic status of Cyrtodactylus khasiensis tamaiensis (Smith, 1940) and description of a new species allied to Cyrtodactylus chrysopylos Bauer, 2003 from Myanmar (Reptilia: Gekkonidae). Hamadryad 34 (1): 62-74.

Mathew R. 2007. Reptilia. In: Director (ed.) State Fauna. Series 14. Fauna of Mizoram 545-578. Zoological Survey of India, Calcutta.

Minh B., Schmidt H., Chernomor O., Schrempf D., Woodhams M., von Haeseler A. \& Lanfear R. 2020. IQ-TREE 2: New models and efficient methods for phylogenetic inference in the genomic era. Molecular Biology and Evolution 37:1530-1534. https://doi.org/10.1093/molbev/msaa015

Mirza Z.A., Bhosale H., Ansari F., Phansalkar P., Sawant M., Gowande G. \& Patel H. 2021. A new species of geckos of the genus Cyrtodactylus Gray, 1827 from Arunachal Pradesh, India. Evolutionary Systematics 5: 13-23. https://doi.org/10.3897/evolsyst.5.61667

Muansanga L., Decemson Ht., Biakzuala L., Hmar G.Z., Lalremsanga H.T., Das M. \& Purkayastha J. 2020. First Record of the Jampui Bent-toed Gecko, Cyrtodactylus montanus Agarwal, Mahony, Giri, Chaitanya, and Bauer 2018 (Squamata: Gekkonidae), from Mizoram, India. Reptiles \& Amphibians 27 (2): 267-268. https://doi.org/10.17161/randa.v27i2.14325

Pawar S. \& Birand A. 2001. A survey of amphibians, reptiles, and birds in Northeast India. CERC Technical Report \#6, Centre for Ecological Research and Conservation, Mysore.

Purkayastha J., Das M., Bohra S.C., Bauer A.M. \& Agarwal I. 2020. Another new Cyrtodactylus (Squamata: Gekkonidae) from Guwahati, Assam, India. Zootaxa 4732 (3): 375-392.

https://doi.org/10.11646/zootaxa.4732.3.2

Purkayastha J., Lalremsanga H.T., Bohra S.C., Biakzuala L., Decemson H., Muansanga L., Vabeiryureilai M., Chauhan S. \& Rathee Y.S. 2021. Four new Bent-toed geckos (Cyrtodactylus Gray: Squamata: Gekkonidae) from Northeast India. Zootaxa 4980 (3): 451-489.

https://doi.org/10.11646/zootaxa.4980.3.2

Sabaj M.H. 2020. Codes for Natural History Collections in Ichthyology and Herpetology. Copeia 108 (2): 593-699. https://doi.org/10.1643/ASIHCODONS2020

Tamura K. \& Nei M. 1993. Estimation of the number of nucleotide substitutions in the control region of mitochondrial DNA in humans and chimpanzees. Molecular Biology and Evolution 10 (3): 512-526. https://doi.org/10.1093/oxfordjournals.molbev.a040023 
TordoffA.W., Duckworth J.W., Macfarlane C., Ravn M. \& Tallant J. 2020. Ecosystem profile: Indo-Burma biodiversity hotspot 2020 update. Critical Ecosystem Partnership Fund, Arlington, Virginia.

Uetz P., Freed P. \& Hošek J. 2022. The Reptile Database. Available from: http://www.reptile-database.org [accessed 3 Feb. 2022].

Vogel G., Lalremsanga H.T. \& Vanlalhrima V. 2017. A second species of the genus Blythia Theobald, 1868 (Squamata: Colubridae) from Mizoram, India. Zootaxa 4276 (4): 569-581.

https://doi.org/10.11646/zootaxa.4276.4.8

Vogel G., Nguyen T.V., Lalremsanga H.T., Biakzuala L., Hrima V. \& Poyarkov N.A. 2020. Taxonomic reassessment of the Pareas margaritophorus-macularius species complex (Squamata, Pareidae).Vertebrate Zoology 70 (4): 547-569. https://doi.org/10.26049/VZ70-4-2020-02

Manuscript received: 25 September 2021

Manuscript accepted: 24 November 2021

Published on: 18 February 2022

Topic editor: Tony Robillard

Desk editor: Marianne Salaün

Printed versions of all papers are also deposited in the libraries of the institutes that are members of the EJT consortium: Muséum national d'histoire naturelle, Paris, France; Meise Botanic Garden, Belgium; Royal Museum for Central Africa, Tervuren, Belgium; Royal Belgian Institute of Natural Sciences, Brussels, Belgium; Natural History Museum of Denmark, Copenhagen, Denmark; Naturalis Biodiversity Center, Leiden, the Netherlands; Museo Nacional de Ciencias Naturales-CSIC, Madrid, Spain; Real Jardín Botánico de Madrid CSIC, Spain; Zoological Research Museum Alexander Koenig, Bonn, Germany; National Museum, Prague, Czech Republic. 


\section{Appendix I}

\section{Specimens examined}

\section{Cyrtodactylus aaronbaueri}

Purkayastha, Lalremsanga, Bohra, Biakzuala, Decemson, Muansanga, Vabeiryureilai, Chauhan \& Rathee, 2021

\section{Holotype}

INDIA • $\widehat{O}$ adult; from Durtlang, Aizawl District, Mizoram state; MZMU2015.

\section{Referred material}

INDIA -3 ô adult; from Mizoram University Campus, Aizawl District, Mizoram state; MZMU2012, MZMU2021, MZMU2032 • + adult (MZMU2067), ô adult (MZMU2020); from Tamdil National Wetland Saitual district, Mizoram state - 0 adult (MZMU2014); from Zawngtahlipui stream, Sihphir, Aizawl district, Mizoram state $\bullet 2+q$ adult (MZMU2018, MZMU2056); from Durtlang, Aizawl District, Mizoram state.

\section{Cyrtodactylus agarwali}

Purkayastha, Lalremsanga, Bohra, Biakzuala, Decemson, Muansanga, Vabeiryureilai, Chauhan \& Rathee, 2021

\section{Holotype}

INDIA $\bullet \widehat{O}$ adult; MZMU2160.

\section{Referred material}

INDIA • 3 ô ô adult; (MZMU2157; MZMU2159; MZMU2161), ô sub-adult (MZMU2158); from outskirts of Siju village, South Garo Hills District, Meghalaya state.

\section{Cyrtodactylus bengkhuaiai}

Purkayastha, Lalremsanga, Bohra, Biakzuala, Decemson, Muansanga, Vabeiryureilai, Chauhan \& Rathee, 2021

\section{Holotype}

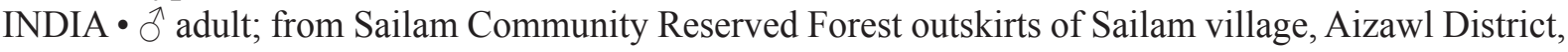
Mizoram state; MZMU1989.

\section{Referred material}

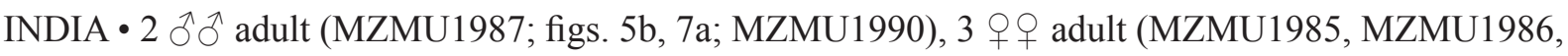
MZMU1988); from Sailam Community Reserved Forest outskirts of Sailam village, Aizawl District, Mizoram state.

\section{Cyrtodactylus guwahatiensis}

Agarwal, Mahony, Giri, Chaitanya \& Bauer, 2018

\section{Referred material}

INDIA $\bullet 5$ के adult (VR/ERS/ZSI/690, VR/ERS/ZSI/691, VR/ERS/ZSI/693, VR/ERS/ZSI/694, VR/ ERS/ZSI/696); 2 + + adult (VR/ERS/ZSI/692, VR/ERS/ZSI/695) from Guwahati, Assam State, India. 


\section{Holotype}

INDIA - ${ }^{7}$; from "Kurseong, Darjeeling dist. (5,000 ft)" [= Kurseong subdivision (ca. $1524 \mathrm{~m}$ asl.), Darjeeling district, West Bengal state, India]; ZSIK 15716.

\section{Referred material}

INDIA • O adult; "Gopaldhara, Darjeling dist." $[=$ Gopaldhara Tea Estate, near Mirik town, Darjeeling district, West Bengal state, India]; ZSIK 19546.

\section{Cyrtodactylus karsticola}

Purkayastha, Lalremsanga, Bohra, Biakzuala, Decemson, Muansanga,

Vabeiryureilai, Chauhan \& Rathee, 2021

\section{Holotype}

INDIA $\bullet \hat{\jmath}$ adult; MZMU2155.

\section{Referred material}

INDIA • 2 $\widehat{\partial}$ adult (MZMU2154, MZMU2156), $q$ adult (MZMU2153); from outskirts of Siju village, South Garo Hills District, Meghalaya state.

\section{Cyrtodactylus khasiensis (Jerdon, 1870)}

\section{Paralectotypes}

INDIA • + adult (ZSIK 6199), $\delta$ adult (ZSIK 6197); collected along with lectotype.

\section{Referred material}

INDIA • $\widehat{\jmath}$ adult (ZSIK 5831, ZSIK 5832), $q$ adult (ZSIK 5828); from "Cherrapunjee" [now Sohra town, East Khasi Hills district, Meghalaya state, India].

\section{Cyrtodactylus urbanus}

Purkayastha, Das, Bohra, Bauer \& Agarwal, 2020

\section{Holotype}

INDIA $\bullet$ ô adult; VR/ERS/ZSI/683.

\section{Referred material}

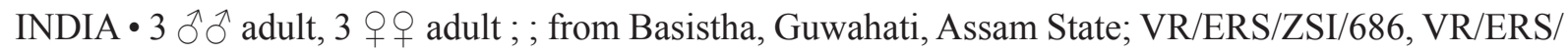
ZSI/685, VR/ERS/ZSI/687, VR/ERS/ZSI/684, VR/ERS/ZSI/688, VR/ERS/ZSI/689. 